\title{
HOMOLOGICAL ALGEBRA AND THE EILENBERG-MOORE SPECTRAL SEQUENCE
}

\author{
BY \\ LARRY SMITH( $\left.{ }^{1}\right)$
}

In [6] Eilenberg and Moore have developed a spectral sequence of great use in algebraic topology. To give a brief description suppose that $\xi_{0}=\left(E_{0}, p_{0}, B_{0}, F\right)$ is a Serre fibre space, $B_{0}$ is simply connected and $f: B \rightarrow B_{0}$ is a continuous map. We then can form the diagram

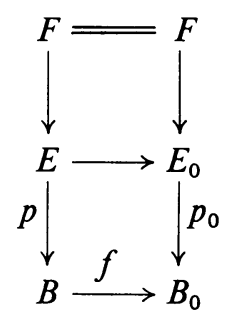

where $\xi=(E, p, B, F)$ is the induced fibre space. Eilenberg and Moore [6] have constructed a spectral sequence $\left\{E_{r}, d_{r}\right\}$ with

(i) $E_{r} \Rightarrow H^{*}(E ; k)$,

(ii) $E_{2}=\operatorname{Tor}_{H^{*}\left(B_{0} ; k\right)}\left(H^{*}(B ; k), H^{*}\left(E_{0} ; k\right)\right)$, where $k$ is a field.

In Part I we shall give a short summary of how one constructs this spectral sequence. Various elementary properties are developed.

In Part II we develop some simple devices to compute $\operatorname{Tor}_{\Lambda}(A, B)$ when $\Lambda$ is a polynomial algebra. These results while basically not new are spread throughout the literature. This material owes much to Borel and the presentation here is based on ideas of J. Moore and P. Baum. These algebraic considerations lead us to a collapse theorem for the spectral sequence in several situations of geometric interest. We close with some applications.

This is a portion of the author's doctoral dissertation completed under the direction of Professor W. S. Massey, whom we wish to thank for much useful guidance. We also wish to thank J. P. May, P. F. Baum and E. O'Neil for useful discussions and suggestions.

\section{Part I. The Eilenberg-Moore Spectral Sequence}

In the fundamental paper [13] John Moore introduced a new type of homological algebra and indicated some of its ramifications in topology. The spectral sequence

Received by the editors August 2, 1966.

(1) Partially supported by NSF-GP-4037 and NSF-GP-3946. 
developed in $\$ 3$ below is due to Eilenberg-Moore [6] and is a natural outgrowth of the work in [13]. We will begin by reviewing the requisite homological algebra to construct this spectral sequence. Applications to topology will appear in the second part. (See also [2], [6], [17].)

1. Differential homological algebra. In this section we shall develop the homological algebra that we shall need later. We shall assume that the reader is familiar with the homological algebra section of [1]. While differential homological algebra has appeared in print before (see [2], [5], [10], [13]) it will be convenient to give a connected account, building up the results needed for the applications. The material below is due to J. Moore.

Throughout this chapter we will be working over a fixed commutative ring $k$, called the ground ring. Our notation and terminology will be that of [10], $\otimes$ means $\otimes_{k}$.

Let $\Lambda$ be a connected differential $k$-algebra. By a $\Lambda$-module we shall mean a left differential $\Lambda$-module. A morphism of $\Lambda$-modules will mean a differential morphism of degree 0 . This describes the category of left differential $\Lambda$-modules, which we denote by $\mathscr{L} \mathscr{D} \mathscr{M} / \Lambda$. If $M$ is an object of $\mathscr{L} \mathscr{D} \mathscr{M} / \Lambda$ we denote this fact by ${ }_{\Lambda} M$. A sequence of $\Lambda$-modules

$$
\rightarrow M^{n-1} \rightarrow M^{n} \rightarrow M^{n+1} \rightarrow
$$

is said to be proper exact if the three sequences

$$
\begin{aligned}
& \rightarrow\left(M^{n-1}\right)^{\#} \rightarrow\left(M^{n}\right)^{\#} \rightarrow\left(M^{n+1}\right)^{\#} \rightarrow \\
& \rightarrow Z\left(M^{n-1}\right) \rightarrow Z\left(M^{n}\right) \rightarrow Z\left(M^{n+1}\right) \rightarrow \\
& \rightarrow H\left(M^{n-1}\right) \rightarrow H\left(M^{n}\right) \rightarrow H\left(M^{n+1}\right) \rightarrow
\end{aligned}
$$

are exact sequences of graded $k$-modules. (Here $M^{\#}$ denotes the underlying graded $k$-module of the $\Lambda$-module $M$.)

If $\cdots \rightarrow M^{n-1} \rightarrow M^{n} \rightarrow M^{n+1} \rightarrow \cdots$ is a sequence of $\Lambda$-modules then each $M^{n}$ is a $\Lambda$-module and we denote by $M^{n, q}$ the homogeneous component of $M^{n}$ of degree $q$.

A proper resolution of a $\Lambda$-module $M$ is a sequence of $\Lambda$-modules

$$
\longrightarrow M^{n-1} \stackrel{d_{E}^{n-1}}{\longrightarrow} M^{n} \stackrel{d_{E}^{n}}{\longrightarrow} \cdots \longrightarrow M^{0} \stackrel{\varepsilon}{\longrightarrow} M \longrightarrow 0
$$

indexed by the nonpositive integers, that is proper exact.

If $(\mathrm{X})$ is a proper resolution of $M$ we can form the bigraded $k$-module $\left\{M^{n, q}\right\}$. If we denote by $d_{I}$ the internal differential $d_{I}^{n, q}: M^{n, q} \rightarrow M^{n, q+1}$ and by $d_{E}$ the resolution differential then we readily see that the collection $\left\{M^{n, q}, d_{I}^{n, q}, d_{E}^{n, q}\right\}$ is a bicomplex in the sense of [10]. The associated graded object $D(X)=\operatorname{Tor}(X)$ is easily seen to be a differential $\Lambda$-module. We shall refer to $q$ as the internal degree and $n$ as the external or homological degree. 
A differential $\Lambda$-module $P$ is called a proper projective $\Lambda$-module, if every diagram of $\Lambda$-modules and morphisms

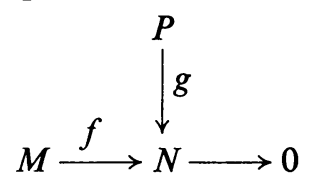

where $f$ is a proper epic, can be completed to a commutative diagram

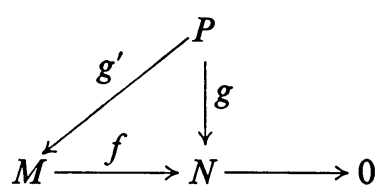

by a map of $\Lambda$-modules $g^{\prime}$.

In [10, XII, Lemma 11.5] it is shown that the category of differential $k$-modules has enough proper projectives, i.e. given any differential $k$-module $M$, there exists a proper projective $k$-module $V$, and a proper epic

$$
V \stackrel{\varepsilon}{\longrightarrow} M \longrightarrow 0 \text {. }
$$

If $V$ is a proper projective $k$-module then $\Lambda \otimes V$ is a proper projective $\Lambda$-module, and every proper projective $\Lambda$-module is a direct summand of such a gadget. Thus the category of $\Lambda$-modules has enough proper projectives, for given ${ }_{\Lambda} M$ choose a proper projective $k$-module with a proper epic $\varepsilon^{\prime}: V \rightarrow M^{\#}$, then

$$
P=\Lambda \otimes V \stackrel{\varepsilon^{\prime}}{\longrightarrow} \Lambda \otimes M \longrightarrow M \longrightarrow 0
$$

provides us with a proper projective $\Lambda$-module $P$ and a proper epic $\varepsilon: P \rightarrow M$. We can now proceed in the standard fashion to form a $\Lambda$-proper projective resolution of $M$.

The definition of proper projective is motivated by the observation that if $X \rightarrow M$ is a $\Lambda$-proper projective resolution of $M$ then $H_{I}(X) \rightarrow H(M)$ is a projective resolution of $H(M)$ as an $H(\Lambda)$-module. (Here $H_{I}(X)=H\left(X, d_{I}\right)$, i.e. as remarked above $X$ is a bicomplex .with respect to the internal differential $d_{I}$ and the external differential $d_{E}$, and $H_{I}(X)$ is the homology of $X$ with respect to $d_{I}$.)

Right differential $\Lambda$-modules are treated analogously. The category of right differential $\Lambda$-modules is denoted by $\mathscr{R} \mathscr{D} \mathscr{M} / \Lambda$.

Suppose that $\left(N_{\Lambda},{ }_{\Lambda} M\right)$ are given and that $X \rightarrow M$ and $Y \rightarrow N$ are proper projective $\Lambda$-resolutions. Recall that $D(X)$ is a differential $\Lambda$-module with differential $d=d_{I}+d_{E}$, and similarly for $D(Y)$.

Definition 1.1. $\operatorname{Tor}_{\Lambda}(N, M)=H\left[D(X) \otimes_{\Lambda} D(Y)\right]$.

One readily verifies that $\operatorname{Tor}_{\Lambda}(N, M)$ is independent of the particular resolutions chosen and that

$$
\operatorname{Tor}_{\Lambda}(N, M)=H\left[D(Y) \otimes_{\Lambda} M\right]=H\left[N \otimes_{\Lambda} D(X)\right] .
$$


$\operatorname{Tor}_{\Lambda}(N, M)$ is a functor from the product category $\mathscr{R} \mathscr{D} \mathscr{M}|\Lambda \times \mathscr{L} \mathscr{D} \mathscr{M}| \Lambda$ to the category of $k$-modules. If $k=\Lambda$ we obtain the hyperhomology of [10]. If $\Lambda, M, N$ have no differential then $\operatorname{Tor}_{\Lambda}(N, M)$ reduces to the ordinary torsion product of $\Lambda$-modules. Thus Definition 1.1 extends the usual definition of torsion product and justifies the terminology adopted. Note also that $\operatorname{Tor}_{\Lambda}(N, M)$ is a covariant functor of three variables.

LemMA 1.1. Let $\Lambda$ be a differential algebra and suppose given $\left(P_{\Lambda},{ }_{\Lambda} M\right)$ where $P$ is a proper projective $\Lambda$-module. Then

$$
H\left(P \otimes_{\Lambda} M\right)=H(P) \otimes_{H(\Lambda)} H(M) .
$$

Proof. Since every proper projective $\Lambda$-module is a direct summand of a gadget of the form $V \otimes \Lambda$ where $V$ is a proper projective $k$-module it suffices to consider the case $P=V \otimes \Lambda$. Then we have

$$
H\left(P \otimes_{\Lambda} M\right)=H\left(V \otimes \Lambda \otimes_{\Lambda} M\right)=H(V \otimes M)
$$

by [10, XII, Theorem 12.2]; we now obtain

$$
\begin{aligned}
H\left(P \otimes_{\Lambda} M\right) & =H(V) \otimes H(M)=H(V) \otimes H(\Lambda) \otimes_{H(\Lambda)} H(M) \\
& =H(V \otimes \Lambda) \otimes_{H(\Lambda)} H(M)=H(P) \otimes_{H(\Lambda)} H(M)
\end{aligned}
$$

by a second application of [10, XII, Theorem 12.2].

THEOREM 1.2 (EILENBERG-MOORE). Let $\Lambda$ be a differential algebra and $\left(N_{\Lambda},{ }_{\Lambda} M\right)$ be given; then there exists a spectral sequence $\left\{E_{r}, d_{r}\right\}$ such that

(i) $E_{r} \Rightarrow \operatorname{Tor}_{\Lambda}(N, M)$,

(ii) $E_{2}=\operatorname{Tor}_{H(\Lambda)}(H(N), H(M))$.

Proof. Let $X \rightarrow N$ be a proper projective resolution of $N$ as a $\Lambda$-module. Form $D(X)$ and filter it by

$$
F^{-p}[D(X)]^{n}=\sum_{i+j=n ; i \geqq-p} X^{i, j} .
$$

The filtered complex $\left\{F^{-p}[D(X)]\right\}$ then gives rise to a spectral sequence. Because the filtration is not finite in each degree the usual convergence proof does not apply. However, results of [21] show that the limit term of the spectral sequence is independent of the particular resolution $X$ employed. In the special case that $\Lambda$ is simply connected, i.e. $\Lambda^{1}=0$, the resolution constructed in the next section gives rise to a filtered complex that is finite in each degree. Thus in this special case the spectral sequence converges in the usual sense to $\operatorname{Tor}_{\Lambda}(N, M)$. The convergence in the general case is a delicate result and will be proved in [6, II]. By Lemma 1.1 we have

$$
E_{1}=D(H(X)) \otimes_{H(\Lambda)} H(M)
$$

with the differential $d_{1}$ induced by the resolution differential $d_{E}$. Thus

$$
E_{2}=\operatorname{Tor}_{H(\Lambda)}(H(N), H(M)) .
$$


Observe that the spectral sequence of Theorem 1.2 lives in the second quadrant and that $d_{r}$ has bidegree $(r, 1-r)$. This spectral sequence will be called the EilenbergMoore spectral sequence. Observe that the Eilenberg-Moore spectral sequence gives us a decreasing filtration on $\operatorname{Tor}_{\Lambda}(N, M)$ which we will denote by

$$
\left\{F^{-p} \operatorname{Tor}_{\Lambda}^{n}(N, M)\right\}
$$

with the property that

$$
E_{\infty}^{-p, n+p}=F^{-p} \operatorname{Tor}_{\Lambda}^{n}(N, M) / F^{-p+1} \operatorname{Tor}_{\Lambda}^{n}(N, M) .
$$

This spectral sequence is natural in the following sense. Suppose given differential algebras $\Lambda$ and $\Gamma$ and modules $\left(N_{\Lambda},{ }_{\Lambda} M\right)$ and $\left(B_{\Gamma},{ }_{\Gamma} A\right)$ and maps

$$
f: \Lambda \rightarrow \Gamma, g: N \rightarrow B, h: M \rightarrow A
$$

such that

(i) $f$ is a map of differential algebras,

(ii) $g$ and $h$ are maps of differential $k$-modules,

(iii) the diagrams below commute

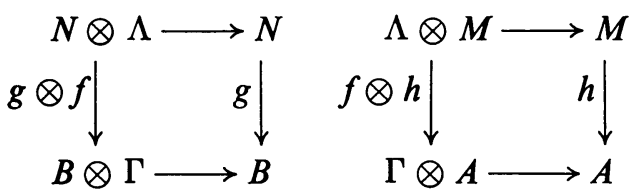

(all this is summarized by saying that $g$ and $h$ are $f$-semilinear). Let $\left\{E_{r}, d_{r}\right\}$ and $\left\{E_{r}^{\prime}, d_{r}^{\prime}\right\}$ be the Eilenberg-Moore spectral sequences for $\left(N_{\Lambda},{ }_{\Lambda} M\right)$ and $\left(A_{\Gamma},{ }_{\Gamma} B\right)$ respectively. Then $f, g$, and $h$ combine to induce a map

$$
\operatorname{Tor}_{f}(g, h):\left\{E_{r}, d_{r}\right\} \rightarrow\left\{E_{r}^{\prime}, d_{r}^{\prime}\right\} .
$$

Corollary 1.3. Let $\Lambda$ and $\Gamma$ be differential algebras and let $\left(N_{\Lambda},{ }_{\Lambda} M\right)$ and $\left(A_{\Gamma},{ }_{\Gamma} B\right)$ be given along with maps

$$
f: \Lambda \rightarrow \Gamma, \quad g: N \rightarrow B, \quad h: M \rightarrow A,
$$

where $g$ and $h$ are $f$-semilinear. If $f, g$, and $h$ induce isomorphisms in homology then

$$
\operatorname{Tor}_{f}(g, h): \operatorname{Tor}_{\Lambda}(N, M) \rightarrow \operatorname{Tor}_{\Gamma}(B, A)
$$

is an isomorphism.

Proof. One merely observes that

$$
\operatorname{Tor}_{f}(g, h)_{2}: E_{2} \rightarrow E_{2}^{\prime}
$$

is an isomorphism.

It will be of considerable use later to have a simple description of the terms $F^{-p} \operatorname{Tor}_{\Lambda}(N, M)$ of the filtration resulting from the Eilenberg-Moore spectral sequence in the special case where $k$ is a field. 
To this end consider the map

$$
\nu: H(N) \otimes H(M) \rightarrow \operatorname{Tor}_{\Lambda}(N, M)
$$

obtained as the composition

$$
H(N) \otimes H(M)=\operatorname{Tor}_{k}(N, M) \rightarrow \operatorname{Tor}_{\Lambda}(N, M) .
$$

Proposition 1.4. $F^{0} \operatorname{Tor}_{\Lambda}(N, M)=\operatorname{im} \nu$ when $k$ is a field.

Proof. Let $\left\{E_{r}, d_{r}\right\}$ be the Eilenberg-Moore spectral sequence for the situation $\left(N_{\Lambda},{ }_{\Lambda} M\right)$ and $\left\{\bar{E}_{r}, \bar{d}_{r}\right\}$ the Eilenberg-Moore spectral sequence for the situation $\left(N_{k},{ }_{k} M\right)$. Then we have a map of spectral sequences

$$
\phi_{r}=\operatorname{Tor}_{n}(1,1)_{r}: \bar{E}_{r} \rightarrow E_{r}
$$

notice that, since $k$ is a field,

$$
\bar{E}_{2}=\operatorname{Tor}_{k}(H(N), H(M))=H(N) \otimes H(M),
$$

i.e.,

(\#)

$$
E_{2}^{0, *}=H(N) \otimes H(M), \quad E_{2}^{-p, *}=0, \quad p \neq 0 .
$$

Now $\phi_{2}: \bar{E}_{2} \rightarrow E_{2}$ is given by

$$
\phi_{2}: H(N) \otimes H(M) \rightarrow H(N) \otimes_{H(\Lambda)} H(M)=E_{2}^{0, *},
$$

which is the natural epimorphism.

It follows from (\#) that $\bar{E}_{2}=\bar{E}_{\infty}$. Using the edge homomorphism [10, XI.1] we see that

$$
\phi: F^{0} \operatorname{Tor}_{k}(N, M) \rightarrow \operatorname{Tor}_{\Lambda}(N, M)
$$

is onto. Since $F^{0} \operatorname{Tor}_{k}(N, M)=\bar{E}_{\infty}^{0, *}=H(N) \otimes H(M)$ we see that the result follows.

Since $v$ vanishes on elements of the form

$$
x a \otimes y-x \otimes a y
$$

$x \in H(N), a \in H(\Lambda), y \in H(M)$ we see that $\nu$ defines a map

$$
\nu^{\prime}: H(N) \otimes_{H(\Lambda)} H(M) \rightarrow \operatorname{Tor}_{\Lambda}(N, M)
$$

and we have

Proposition 1.4'. If $k$ is a field then $F^{0} \operatorname{Tor}_{\Lambda}(N, M)=\operatorname{im} \nu^{\prime}$.

To obtain a characterization of $F^{-1} \operatorname{Tor}_{\Lambda}(N, M)$ we will find it useful to introduce some technical tools.

2. The bar construction. Throughout this section the ground ring $k$ will be a field.

We will suppose given a connected, simply connected (i.e. $\Lambda^{1}=0$ ) differential $k$-algebra $\Lambda$. Let $M$ be a right $\Lambda$-module. We will define a specific proper projective resolution of $M$ over $\Lambda$ called the bar construction and denoted by $B(\Lambda, M)$. 
Define

$$
B^{-n}(\Lambda, M)=\Lambda \otimes \bar{\Lambda} \otimes \cdots \otimes \bar{\Lambda} \otimes M,
$$

$\bar{\Lambda}=\{a \in \Lambda \mid \operatorname{deg} a>0\}$.

Next define

by the usual formula

$$
d_{E}: B^{-n}(\Lambda, M) \rightarrow B^{-n+1}(\Lambda, M)
$$

where $s(i)$ is given by

$$
\begin{aligned}
d_{E}\left(a\left[a_{1}|\cdots| a_{n}\right] x\right)= & a a_{1}\left[a_{2}|\cdots| a_{n}\right] x \\
& +\sum(-1)^{s(i)} a\left[a_{1}|\cdots| a_{i} a_{i+1}|\cdots| a_{n}\right] x \\
& +(-1)^{s(n-1)} a\left[a_{1}|\cdots| a_{n-1}\right] a_{n} x
\end{aligned}
$$

$$
s(i)=\operatorname{deg} a+\sum_{j \leq 1} \operatorname{deg} a_{j}-i .
$$

A specific contracting homotopy for $B(\Lambda, M)$ is given by

$$
\begin{aligned}
s_{E}\left(a\left[a_{1}|\cdots| a_{n}\right] x\right) & =a\left[a_{1}|\cdots| a_{n}\right] x \quad \text { if } \operatorname{deg} a>0, \\
& =0 \quad \text { if } \operatorname{deg} a=0 .
\end{aligned}
$$

It is well known [1, II.2] or [10, XI.2] that $B(\Lambda, M)$ is acyclic with homology $M$.

Note also that, since $\Lambda$ is simply connected, $B(\Lambda, M)$ is of finite type.

WARNING. The bigrading on $B(\Lambda, M)$ is not the usual grading. It differs in that the homological degree is graded on the nonpositive integers instead of the usual procedure of using the nonnegative integers.

We now give each $B^{-n}(\Lambda, M)$ the structure of a differential $\Lambda$-module by defining

by

$$
d_{I}: B^{-n}(\Lambda, M) \rightarrow B^{-n}(\Lambda, M)
$$

$$
\begin{aligned}
d_{I}\left(a\left[a_{1}|\cdots| a_{n}\right] x\right)= & d a\left[a_{1}|\cdots| a_{n}\right] x \\
& +\sum(-1)^{s(i-1)} a\left[a_{1}|\cdots| d a_{i}|\cdots| a_{n}\right] x \\
& +(-1)^{s(n-1)} a\left[a_{1}|\cdots| a_{n}\right] d_{M} x
\end{aligned}
$$

where $d: \Lambda \rightarrow \Lambda$ and $d_{M}: M \rightarrow M$ are the differentials in $\Lambda$ and $M$ respectively. $B^{-n}(\Lambda, M)$ is graded by

$$
\operatorname{deg}_{I}\left(a\left[a_{1}|\cdots| a_{n}\right] x\right)=\operatorname{deg} a+\sum \operatorname{deg} a_{i}+\operatorname{deg} b
$$

and $B^{-n}(\Lambda, M)$ is given a $\Lambda$-module structure by

$$
a^{\prime}\left(a\left[a_{1}|\cdots| a_{n}\right] x\right)=a^{\prime} a\left[a_{1}|\cdots| a_{n}\right] x
$$

for all $a^{\prime}, a, a_{i} \in \Lambda$ and $x \in M$. It is now straightforward to verify that each $B^{-n}(\Lambda, M)$ is a differential $\Lambda$-module and that $d_{E}$ is a map of differential $\Lambda$-modules.

Leмma 2.1. If $k$ is a field then every differential $k$-module is a proper projective $k$-module.

Proof. This follows directly from [10, XII, Lemma 11.4]. 
LEMMA 2.2. $B^{-n}(\Lambda, M)$ is a proper projective $\Lambda$-module.

Proof. By definition

$$
B^{-n}(\Lambda, M)=\Lambda \otimes \bar{\Lambda} \otimes \cdots \otimes \bar{\Lambda} \otimes M
$$

as a $\Lambda$-module. Since $[\bar{\Lambda} \otimes \cdots \otimes \bar{\Lambda} \otimes M]$ is a proper projective $k$-module by Lemma 2.1 it follows that $B^{-n}(\Lambda, M)$ is a proper projective $\Lambda$-module.

Let $Z_{I} B^{-n}(\Lambda, M)$ denote the cycles in $B^{-n}(\Lambda, M)$ under the differential $d_{I}$.

Lemma 2.3. $s_{E}\left(Z_{I} B^{-n}(\Lambda, M)\right) \subset Z_{I} B^{-n-1}(\Lambda, M)$.

Proof. By direct computation.

LEMMA 2.4. The sequence

is exact.

$$
\longrightarrow Z_{I} B^{-n}(\Lambda, M) \stackrel{d_{E}}{\longrightarrow} Z_{I} B^{-n+1}(\Lambda, M) \longrightarrow
$$

Proof. Direct from Lemma 2.3 and the fact that

$$
s_{E} d_{E}+d_{E} s_{E}=1 \text {. }
$$

Proposition 2.5. The complex $B(\Lambda, M)$ is a proper projective resolution of $M$ as a $\Lambda$-module.

Proof. From Lemmas $2.2-2.4$ we see that it only remains to show that the sequence

is exact.

$$
\cdots \rightarrow H_{I}\left(B^{-n}(\Lambda, M)\right) \rightarrow H_{I}\left(B^{-n+1}(\Lambda, M)\right) \rightarrow \cdots
$$

Since $k$ is a field the Künneth theorem is valid over $k$ and so we see that

$$
\begin{aligned}
H_{I}\left(B^{-n}(\Lambda, M)\right) & =H(\Lambda) \otimes H(\bar{\Lambda}) \otimes \cdots \otimes H(\bar{\Lambda}) \otimes H(M) \\
& =B^{-n}(H(\Lambda), H(M)),
\end{aligned}
$$

which is just the bar construction on $H(M)$ over $H(\Lambda)$. Since we already know this is exact, the result follows.

If $N$ is a $\Lambda$-module then we can form $N \otimes_{\Lambda} B(\Lambda, M)$, which we will denote by $\mathscr{X}$. We observe that Proposition 2.5 implies that $H(\mathscr{X})=\operatorname{Tor}_{\Lambda}(N, M)$.

We have

$$
\mathscr{X}^{-n}=N \otimes \bar{\Lambda} \otimes \cdots \otimes \bar{\Lambda} \otimes M
$$

where

and

$$
\begin{aligned}
d_{E}\left(y\left[a_{1}|\cdots| a_{n}\right] x\right)= & y a_{1}\left[a_{2}|\cdots| a_{n}\right] x \\
& +\sum(-1)^{s(i)} y\left[a_{1}|\cdots| a_{i} a_{i+1}|\cdots| a_{n}\right] x \\
& +(-1)^{s(n-1)} y\left[a_{1}|\cdots| a_{n-1}\right] a_{n} x
\end{aligned}
$$

$$
\begin{aligned}
d_{l}\left(y\left[a_{1}|\cdots| a_{n}\right] x\right)= & d_{N} y\left[a_{1}|\cdots| a_{n}\right] x \\
& +\sum(-1)^{s(i-1)} y\left[a_{1}|\cdots| d a_{i}|\cdots| a_{n}\right] x \\
& +(-1)^{s(n-1)} y\left[a_{1}|\cdots| a_{n}\right] d_{M} x,
\end{aligned}
$$

where $d_{N}, d, d_{M}$ are the differentials in $N, \Lambda, M$ respectively. 
The Eilenberg-Moore spectral sequence of $\left(N_{\Lambda},{ }_{\Lambda} M\right)$ is obtained by filtering $\mathscr{X}$ by

$$
F^{-p} \mathscr{X}=\left\{y\left[a_{1}|\cdots| a_{n}\right] x \mid n \leqq p\right\} .
$$

If $y\left[a_{1}|\cdots| a_{n}\right] \in \mathscr{X}$ then its bidegree is given by

$$
\operatorname{bideg}\left(y\left[a_{1}|\cdots| a_{n}\right] x\right)=\left(-n, \operatorname{deg} y+\sum \operatorname{deg} a_{i}+\operatorname{deg} x\right) .
$$

As usual the first degree is called the external or homological degree and the second degree the internal degree.

We are now ready to obtain a description of $F^{-1} \operatorname{Tor}_{\Lambda}(N, M)$. To this end consider the map

$$
\phi: H(N) \otimes \overline{H(\Lambda)} \otimes H(M) \rightarrow H(N) \otimes H(M)
$$

defined by

$$
\phi(y \otimes a \otimes x)=y a \otimes x-y \otimes a x .
$$

We are going to define two maps

$$
\begin{aligned}
T: \operatorname{ker} \phi & \rightarrow \operatorname{Tor}_{H(\Lambda)}^{-1 *}(H(N), H(M)), \\
T^{\#}: \operatorname{ker} \phi & \rightarrow F^{-1} \operatorname{Tor}_{\Lambda}(N, M) .
\end{aligned}
$$

We begin by defining $T$. Let (see [1, II.2])

$$
v=\sum y_{i} \otimes a_{i} \otimes x_{i}
$$

by an element of $\operatorname{ker} \phi$. Then

$$
\sum y_{i} a_{i} \otimes x_{\mathfrak{i}}-y_{i} \otimes a_{\mathfrak{i}} x_{\mathfrak{i}}=0 ;
$$

therefore $w=\sum y_{i}\left[a_{i}\right] x_{i}$ is a cycle in the complex $\mathscr{X}$, and hence determines an element

$$
\operatorname{cls}(w) \in \operatorname{Tor}_{H(\Lambda)}^{-1} *(H(N), H(M))
$$

and it is immediate that we have

Lemma 2.6. Define $T: \operatorname{Ker} \phi \rightarrow \operatorname{Tor}_{H(\Lambda)}^{-1}(H(N), H(M))$ by $v \rightarrow \operatorname{cls}(w)$ as above. Then $T$ is an epimorphism of vector spaces.

To define $T^{\#}$ let $v=\sum y_{i} \otimes a_{i} \otimes x_{i} \in \operatorname{ker} \phi$. Choose representative cocycles $\bar{y}_{i} \in N$, $\bar{a}_{i} \in \Lambda, \bar{x}_{i} \in M$ for $y_{i}, a_{i}, x_{i}$ respectively. Then, since $\phi(v)=0$, there exists an element $U \in N \otimes M$ such that

$$
\sum \bar{y}_{i} \bar{a}_{i} \otimes \bar{x}_{i}-\bar{y}_{i} \otimes \bar{a}_{i} \bar{x}_{i}=d^{\otimes}(U),
$$

where $d^{\otimes}$ is the usual differential on the tensor product $N \otimes M$. Consider the element

$$
z=\sum \bar{y}_{i}\left[\bar{a}_{i}\right] \bar{x}_{i}-U \in \mathscr{X} .
$$

Direct computation shows that $d(z)=0, d$ the differential on $\mathscr{X}$. Hence $z \in \mathscr{X}$ is a cycle and determines an element

$$
\text { cls }(z) \in H(\mathscr{X}, d)=\operatorname{Tor}_{\Lambda}(N, M) .
$$


It is clear from the construction that cls $(z)$ has filtration degree -1 .

There are two kinds of indeterminacy arising from the choices that we made in constructing cls $(z)$. The first arises from the choice of the class $U \in N \otimes M$. So suppose that $U^{\prime} \in N \otimes M$ is another class with

$$
d^{\otimes}\left(U^{\prime}\right)=\sum \bar{y}_{i} \bar{a}_{i} \otimes \bar{x}_{i}-\bar{y}_{i} \otimes \bar{a}_{i} \bar{x}_{i}
$$

then $U-U^{\prime}$ is a cycle in $N \otimes M$. Therefore we see that cls $(z)$ will be determined modulo

$$
\operatorname{im}\left\{H(N) \otimes H(M) \rightarrow \operatorname{Tor}_{\Lambda}(N, M)\right\}=F^{0} \operatorname{Tor}_{\Lambda}(N, M) .
$$

The second ambiguity arises from the original choice of the cycles $\bar{y}_{i}, \bar{a}_{i}, \bar{x}_{i}$. To handle the indeterminacy arising from these choices we make the following observation. Suppose that $\bar{a} \in \mathscr{X}$ and $\bar{x} \in M$ are cycles and that $\bar{y} \in N$ is arbitrary, then

$$
d(\bar{y}[\bar{a}] \bar{x})=d_{N} \bar{y}[\bar{a}] \bar{x}+\overline{y a}[] \bar{x}-\bar{y}[] \overline{a x}
$$

and therefore $d_{N} \bar{y}[\bar{a}] \bar{x}$ is homologous to $\overline{y a}[] \bar{x}-\bar{y}[] \overline{a x}$ in $\mathscr{X}$. Using this fact and direct computation we can show that the indeterminacy arising from the choices of the classes $\bar{y}_{i}, \bar{a}_{i}, \bar{x}_{i}$ is also contained in

$$
\left.\operatorname{im}\{H(N) \otimes H(M)\} \rightarrow \operatorname{Tor}_{\Lambda}(N, M)\right\}=F^{0} \operatorname{Tor}_{\Lambda}(N, M) .
$$

Therefore if we define $T^{\#}$ by

$$
T^{\#}(v)=\operatorname{cls} z \in F^{-1} \operatorname{Tor}_{\Lambda}(N, M),
$$

then in the terminology of [10] we may regard $T^{\#}$ as an additive relation

$$
T^{\#}: H(N) \otimes \overline{H(\Lambda)} \otimes H(M) \rightarrow F^{-1} \operatorname{Tor}_{\Lambda}(N, M)
$$

or as a homomorphism of vector spaces

$$
T^{\#}: \operatorname{ker} \phi \rightarrow F^{-1} \operatorname{Tor}_{\Lambda}(N, M) / F^{0} \operatorname{Tor}_{\Lambda}(N, M)=E_{\infty}^{-1, *} .
$$

The interpretation of $T^{\#}$ as an additive relation will prove most useful later in the topological applications and therefore we emphasize this point of view.

Proposition 2.7. The elements $\left\{T^{\#}(v) \mid v \in \operatorname{ker} \phi\right\}$ generate $F^{-1} \operatorname{Tor}_{\Lambda}(N, M)$ as a vector space.

Proof. Let $\left\{E_{r}, d_{r}\right\}$ denote the Eilenberg-Moore spectral sequence for the situation $\left(N_{\Lambda},{ }_{\Lambda} M\right)$. We then have an exact sequence of $k$-modules

$$
0 \longrightarrow E_{\infty}^{0, *}=F^{0} \operatorname{Tor}_{\Lambda}(N, M) \longrightarrow F^{-1} \operatorname{Tor}_{\Lambda}(N, M) \stackrel{\sigma}{\longrightarrow} E_{\infty}^{-1, *} \longrightarrow 0 .
$$

Since the indeterminacy of $T^{\#}$ is $F^{0} \operatorname{Tor}_{\Lambda}(N, M)$, it clearly suffices to show that the elements $\left\{T^{\#}(v) \mid v \in \operatorname{ker} \phi\right\}$ generate $F^{-1} \operatorname{Tor}_{\Lambda}(N, M) / F^{0} \operatorname{Tor}_{\Lambda}(N, M)=E_{\infty}^{-1, *}$ as a $k$-module.

To this end we note that

$$
d_{r}: E_{r}^{-1, *} \rightarrow 0
$$

because

$$
d_{r}\left(E_{r}^{-1, *}\right) \subset E_{r}^{r-1, *}=0, \quad r \geqq 2 .
$$


Therefore we can identify $E_{r}^{-1, *}$ with a quotient module of $E_{2}^{-1, *}$ and so each element $W \in E_{2}^{-1, *}$ determines an element $W_{\infty}$, possibly zero, of $E_{\infty}^{-1, *}$.

Now since $\{T(v) \mid v \in \operatorname{ker} \phi\}$ generates $\operatorname{Tor}_{H(\Lambda)}^{-1}(H(N), H(M))=E_{2}^{-1, *}$ as a vector space it follows that $\left\{T(v)_{\infty} \mid v \in \operatorname{ker} \phi\right\}$ generates $E_{\infty}^{-1, *}$ as a vector space.

Thus the proof will be complete if we can show

$$
\sigma\left(T^{\#}(v)\right)=T(v)_{\infty} .
$$

But this is just a direct calculation. Using the notation preceding the proposition, set

$$
z=\sum \bar{y}_{i}\left[\bar{a}_{i}\right] \bar{x}_{i}-U \in \mathscr{X}
$$

then $z$ determines an element of $E_{0}$, which we denote by $z^{\prime}$, and note that

$$
z^{\prime}=\sum \bar{y}_{i}\left[\bar{a}_{i}\right] \bar{x}_{i} \in E_{0}
$$

and is a $d_{I}=d_{0}$ cycle. Then in the obvious notation we have

$$
\operatorname{cls}_{I}\left(z^{\prime}\right)=\sum y_{i}\left[a_{i}\right] x_{i} \in E_{1}=H(N) \otimes_{H(\Lambda)} B(H(\Lambda), H(M))
$$

and $\operatorname{cls}_{I}\left(z^{\prime}\right)$ is readily seen to be a $d_{E}=d_{1}$ cycle, and so we have

Hence it follows that

$$
\operatorname{cls}_{E} \operatorname{cls}_{I}\left(z^{\prime}\right)=T(v) \text {. }
$$

$$
\sigma T^{\#}(v)=T(v)_{\infty}
$$

as claimed, and the proposition is proved.

It would be of great value for the geometric applications to have an analogous description of the other terms $F^{-p} \operatorname{Tor}_{\Lambda}(N, M)$ (see $\S 4$ below).

3. Geometry. In this section we will begin to apply the results of the previous section to geometry. These results are due to Eilenberg-Moore [6].

By a topological space we shall always mean a topological space of the homotopy type of a countable cw-complex whose integral homology is of finite type. All spaces will come furnished with basepoints. By a fibration we shall mean a fibration in the sense of Serre. We recall that for such fibrations we can always form the induced fibration. We turn to this now.

Suppose that we are given a diagram

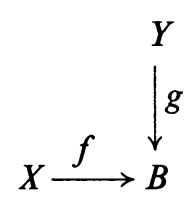

where $g: Y \rightarrow B$ is a fibre map. We define

$$
X \times_{B} Y=\{(x, y) \in X \times Y \mid f(x)=g(y)\}
$$

(this rather suggestive notation is due to P. Baum) and define

$$
\begin{array}{ll}
\bar{g}: X \times_{B} Y \rightarrow X & \text { by } \bar{g}(x, y)=x, \\
\bar{f}: X \times_{B} Y \rightarrow Y & \text { by } \bar{f}(x, y)=y .
\end{array}
$$


Then we have a commutative diagram

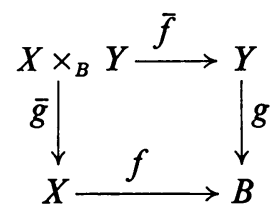

(note that $\bar{f}$ maps the fibre homeomorphically).

We now show how the work of the previous sections can be applied to the problem of computing the cohomology of the space $X \times_{B} Y$.

Throughout this section we shall assume given a fixed diagram

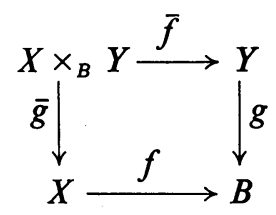

where $B$ is simply connected. The fibre of the fibre maps $g$, and $\bar{g}$ will be denoted by $F$.

For any space $A, C_{*}(A)$ will denote the normalized singular chain complex of $A$ with all vertices at the basepoint. Throughout this section $k$ will be a fixed field, referred to as the ground field. $C^{*}(A)=\operatorname{Hom}\left(C_{*}(A), k\right)$ and $H^{*}(A)=H^{*}(A, k)$.

Note that $C^{*}(A)$ is a connected differential $k$-algebra.

We have a natural map, denoted by $\alpha$

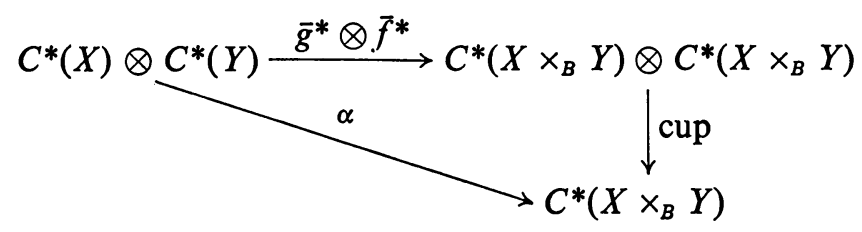

Let

$$
\phi: C^{*}(X) \otimes C^{*}(B) \otimes C^{*}(Y) \rightarrow C^{*}(X) \otimes C^{*}(Y)
$$

by

$$
\phi(u \otimes v \otimes w)=u f^{*}(v) \otimes w-u \otimes g^{*}(v) w
$$

and let

$$
W=\operatorname{im} \phi .
$$

LEMMA 3.1. $\alpha \mid W=0$ and hence $\alpha$ induces $a$ map

$$
\begin{aligned}
& C^{*}(X) \otimes C^{*}(Y) / W \rightarrow C^{*}\left(X \times_{B} Y\right) \\
& \| \\
& C^{*}(X) \otimes_{C^{*}(B)} C^{*}(Y)
\end{aligned}
$$

Proof. Direct calculation. 
Let $P$ be a proper projective resolution of $C^{*}(X)$ as a $C^{*}(B)$ module. Then we have a morphism $\theta$ given by

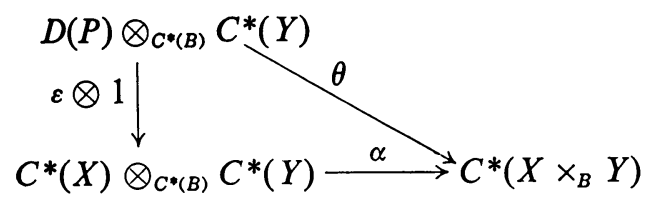

TheOREM 3.2 (EILENBERG-MoORE). The map

$$
\theta: D(P) \otimes_{C^{*}(B)} C^{*}(Y) \rightarrow C^{*}\left(X \times_{B} Y\right)
$$

induces an isomorphism

$$
\theta: \operatorname{Tor}_{C^{*}(B)}\left(C^{*}(X), C^{*}(Y)\right) \rightarrow H^{*}\left(X \times_{B} Y\right) .
$$

Proof. Filter $C^{*}\left(X \times_{B} Y\right)$ by the Serre filtration of the fibring $\bar{g}: X \times_{B} Y \rightarrow X$ and denote the resulting spectral sequence by $\left\{\tilde{E}_{r}, \tilde{d}_{r}\right\}$.

Filter $C^{*}(Y)$ by the Serre filtration of the fibring $g: Y \rightarrow B$ and denote the resulting spectral sequence by $\left\{\bar{E}_{r}, \bar{d}_{r}\right\}$.

Filter $D(P)$ by the total degree and finally filter $D(P) \otimes_{C^{*}(B)} C^{*}(Y)$ by

$$
F^{p}\left[D(P) \otimes_{C^{*}(B)} C^{*}(Y)\right]=\sum_{i+j=p} F^{i} D(P) \otimes_{C^{*}(B)} F^{j}\left(C^{*}(Y)\right)
$$

and denote the resulting spectral sequence by $\left\{E_{r}, d_{r}\right\}$.

Note that all these filtrations are decreasing and complete.

Observe that

$$
\theta: F^{p}\left[d(P) \otimes_{C^{*}(B)} C^{*}(Y)\right] \rightarrow F^{p} C^{*}\left(X \times_{B} Y\right)
$$

and so induces a map of spectral sequences

$$
\left\{\theta_{r}^{*}\right\}:\left\{E_{r}, d_{r}\right\} \rightarrow\left\{\tilde{E}_{r}, \tilde{d}_{r}\right\} .
$$

We will complete the proof by showing that $\theta_{2}^{*}$ is an isomorphism. To this end let us compute $E_{2}$. We may as well assume that $D(P)=V \otimes C^{*}(B)$. Then

$$
E_{0}=V \otimes C^{*}(B) \otimes_{C^{*}(B)} C^{*}(Y)=V \otimes C^{*}(Y)
$$

and

$$
d_{0}(v \otimes y)=v \otimes \bar{d}_{0}(y)
$$

and thus

$$
E_{1}=V \otimes \bar{E}_{1}=V \otimes C^{*}\left(B ; H^{*}(F)\right)
$$

since $B$ is simply connected and all cohomology is of finite type, we can conclude that the local coefficient system $H^{*}(F)$ is trivial and

$$
\begin{aligned}
& E_{1}=V \otimes C^{*}(B) \otimes H^{*}(F)=D(P) \otimes H^{*}(F), \\
& d_{1}=\text { total differential in } D(P)
\end{aligned}
$$


and so by Lemma 3.3 below we have

$$
E_{2}=H[D(P)] \otimes H^{*}(F)=H^{*}(X) \otimes H^{*}(F) .
$$

Now the local coefficient system in the fibration

$$
F \rightarrow X \times_{B} Y \rightarrow Y
$$

is induced from the local coefficient system of the fibration

$$
F \rightarrow Y \rightarrow B,
$$

which is trivial since $B$ is simply connected. Therefore the local coefficient system in

$$
F \rightarrow X \times{ }_{B} Y \rightarrow B
$$

is also trivial. Therefore

$$
E_{2}=H^{*}\left(X ; H^{*}(F)\right)
$$

and the map

$$
H^{*}(X) \otimes H^{*}(F)=E_{2} \stackrel{\theta_{2}^{*}}{\longrightarrow} \widetilde{E}_{2}=H^{*}\left(X: H^{*}(F)\right)
$$

is the usual Künneth map. Since everything in sight is of finite type and the ground ring is a field it follows that this is an isomorphism. Therefore

$$
\left\{\theta_{r}\right\}:\left\{E_{r}, d_{r}\right\} \rightarrow\left\{\tilde{E}_{r}, \tilde{d}_{r}\right\}
$$

is an isomorphism for $r \geqq 2$, which completes the proof.

Lemma 3.3. Given a $\Lambda$-module $M$ and a proper projective resolution $P \rightarrow M$ of $M$ as a $\Lambda$-module, then

$$
H[D(P) ; \text { total } d]=H(M) .
$$

Proof. We note that $\left\{D(P), d_{E}, d_{I}\right\}$ is a double complex and forming its first spectral sequence $[10, \mathrm{XI}$, Theorem 6.1$]$ we obtain a spectral sequence $\left\{E_{r}, d_{r}\right\}$ with

$$
E_{r} \Rightarrow H[D(P)], \quad E_{1}=D(H(M)), \quad d_{1}=d_{E},
$$

and so

$$
E_{2}^{0, *}=H(M), \quad E_{2}^{p, *}=0, \quad p \neq 0
$$

and so $H[D(P)]=H(M)$.

As is well known $H^{*}\left(X \times_{B} Y\right)$ is more than just a $k$-module; it is also an algebra in a natural way. We will now show how to introduce an algebra structure into $\operatorname{Tor}_{C^{*}(B)}\left(C^{*}(X), C^{*}(Y)\right)$.

Proposition 3.4. $\operatorname{Tor}_{C^{*}(B)}\left(C^{*}(X), C^{*}(Y)\right)$ is an algebra in a natural way, and the map

$$
\theta^{*}: \operatorname{Tor}_{C^{*}(B)}\left(C^{*}(X), C^{*}(Y)\right) \rightarrow H^{*}\left(X \times_{B} Y\right)
$$

is an isomorphism of algebras. 
Proof. The Eilenberg-Zilber theorem [10, VIII.8] gives natural chain equivalences

$$
\begin{aligned}
& C^{*}(B \times B) \stackrel{f}{\longrightarrow} C^{*}(B) \otimes C^{*}(B), \\
& C^{*}(X \times X) \stackrel{g}{\longrightarrow} C^{*}(X) \otimes C^{*}(X), \\
& C^{*}(Y \times Y) \stackrel{h}{\longrightarrow} C^{*}(Y) \otimes C^{*}(Y),
\end{aligned}
$$

that are maps of algebras with $g, h$ being $f$-semilinear. Thus by Corollary 1.3 we have an isomorphism

$$
\begin{gathered}
\operatorname{Tor}_{C^{*}(B \times B)}\left(C^{*}(X \times X), C^{*}(Y \times Y)\right) \\
\qquad \operatorname{Tor}_{f}(g, h) \\
\operatorname{Tor}_{C^{*}(B) \otimes C^{*}(B)}\left(C^{*}(X) \otimes C^{*}(X), C^{*}(Y) \otimes C^{*}(Y)\right)
\end{gathered}
$$

Now as in any Künneth situation [5, p. 206] we have a natural map

$$
\begin{array}{r}
\operatorname{Tor}_{C^{*}(B)}\left(C^{*}(X), C^{*}(Y)\right) \otimes \operatorname{Tor}_{C^{*}(B)}\left(C^{*}(X), C^{*}(Y)\right) \\
T \downarrow \\
\quad \operatorname{Tor}_{C^{*}(B) \otimes C^{*}(B)}\left(C^{*}(X) \otimes C^{*}(X), C^{*}(Y) \otimes C^{*}(Y)\right)
\end{array}
$$

Consider the diagonal maps

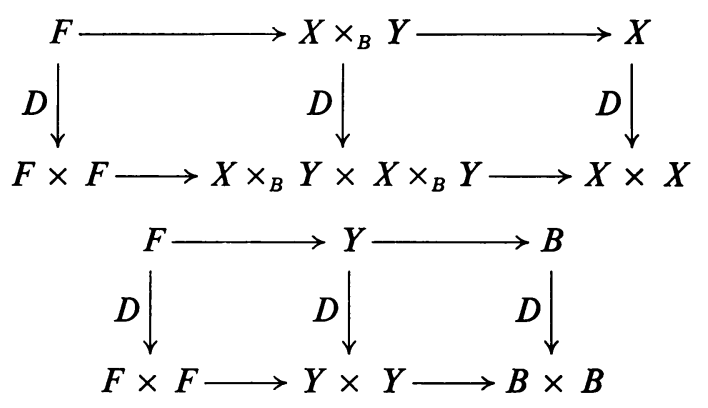

then the composite

$$
\begin{gathered}
\operatorname{Tor}_{C^{*}(B)}\left(C^{*}(X), C^{*}(Y)\right) \otimes \operatorname{Tor}_{C^{*}(B)}\left(C^{*}(X), C^{*}(Y)\right) \\
\operatorname{Tor}_{C^{*}(B) \otimes C^{*}(B)}\left(C^{*}(X) \otimes C^{*}(X), C^{*}(Y) \otimes C^{*}(Y)\right) \\
\operatorname{Tor}_{f}(g, h)^{-1} \downarrow \\
\operatorname{Tor}_{C^{*}(B \times B)}\left(C^{*}(X \times X), C^{*}(Y \times Y)\right) \\
\operatorname{Tor}_{D}(D, D) \downarrow \\
\operatorname{Tor}_{C^{*}(B)}\left(C^{*}(X), C^{*}(Y)\right)
\end{gathered}
$$

is the desired product. 
Remark. Note that the algebra structure in Tor is not a consequence of the existence of algebra structures on $C^{*}(B), C^{*}(X)$ and $C^{*}(Y)$ but of the properties of the Eilenberg-Zilber map and the Künneth theorem.

THEOREM 3.5 (EILENBERG-MOORE). Suppose given a diagram

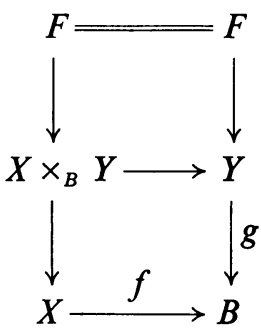

where

(i) $F \rightarrow Y \rightarrow B$ is a Serre fibre space,

(ii) $F \rightarrow X \times_{B} Y \rightarrow X$ is the fibre space induced by the map $f: X \rightarrow B$,

(iii) $B$ is simply connected. Then there exists a spectral sequence of commutative algebras $\left\{E_{r}, d_{r}\right\}$ with

(a) $E_{r} \Rightarrow H^{*}\left(X \times_{B} Y\right)$,

(b) $E_{2}=\operatorname{Tor}_{H^{*}(B)}\left(H^{*}(X), H^{*}(Y)\right)$.

Proof. Form the spectral sequence of Theorem 1.2 for the $C^{*}(B)$-modules $C^{*}(X), C^{*}(Y)$. By Theorem 3.2 it converges to $H^{*}\left(X \times_{B} Y\right)$. A routine examination of the product structure defined in Proposition 3.4 shows that $E_{r}$ is a commutative algebra and that $d_{r}$ is a derivation for $r \geqq 2$.

We shall refer to the spectral sequence of Theorem 3.5 as the Eilenberg-Moore spectral sequence.

A special case of Theorem 3.5 that can not be emphasized enough is the case $X=$ point; for then $X \times_{B} Y=F$ and we obtain

THEOREM 3.6 (EILENBERG-MOORE). Let $F \rightarrow Y \rightarrow B$ be a Serre fibration over the simply connected space $B$. Then there exists a spectral sequence of commutative algebras $\left\{E_{r}, d_{r}\right\}$ with

(a) $E_{r} \Rightarrow H^{*}(F)$,

(b) $E_{2}=\operatorname{Tor}_{H^{*}(B)}\left(k, H^{*}(E)\right)$.

This special case has been used to great advantage by P. Baum in [2].

4. Elementary properties of $E_{\infty}$. In this section we shall develop a description of the terms $E_{\infty}^{0, *}$ and $E_{\infty}^{-1, *}$ along with a description of $F^{-1} H^{*}\left(X \times_{B} Y\right)$ sufficient for the applications later. It would be useful to have an analogous description of the terms $F^{-p} H^{*}\left(X \times_{B} Y\right), p \geqq 2$.

We begin by recalling some basic ideas due to Steenrod [19, §22]. Suppose that $X, Y$ are spaces and that $A \subset X$ is a closed subspace. Suppose further that $f, g: X \rightarrow Y$ are continuous maps and that $f|A=g| A$. We can then define a homomorphism

$$
(f-g)^{*}: H^{*}(Y) \rightarrow H^{*}(X, A)
$$


called the difference homomorphism as follows. Consider the diagram

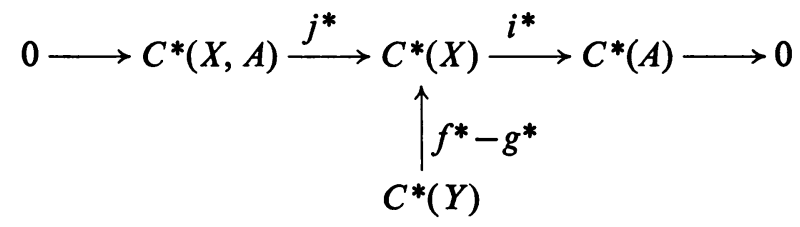

Since $i^{*}\left(f^{*}-g^{*}\right)=0$ and $j^{*}$ is a monomorphism there exists a unique map of chain complexes

$$
(f-g)^{*}: C^{*}(Y) \rightarrow C^{*}(X, A) .
$$

The map this induces in cohomology is called the difference homomorphism and is also denoted by $(f-g)^{*}$.

It will be important to have a more geometric description of $(f-g)^{*}$ which we now proceed to give. (We are indebted to W. S. Massey for this description.)

Let $X \cup_{A} X=X \times\{0\} \cup X \times\{1\} /(a, 0) \sim(a, 1)$ all $a \in A$. Since $f|A=g| A$ it follows that $f$ and $g$ induce a map

$$
f \cup_{A} g: X \cup_{A} X \rightarrow Y
$$

and so we obtain an induced map

$$
\left(f \cup_{A} g\right)^{*}: H^{*}(Y) \rightarrow H^{*}\left(X \cup_{A} X\right) \text {. }
$$

Now let $X_{i}=X \times\{i\}, i=0,1$. Then $X_{i} \subset X \cup_{A} X$ in the obvious fashion. Note that $X_{i}$ is a retract of $X \cup_{A} X$. Thus we have

$$
H^{*}\left(X \cup_{A} X\right)=H^{*}\left(X_{i}\right) \oplus H^{*}\left(X \cup_{A} X, X_{i}\right), \quad i=0,1 ;
$$

further the maps

$$
\varepsilon_{i}:\left(X_{1-i}, A\right) \rightarrow\left(X \cup_{A} X, X_{i}\right), \quad i=0,1,
$$

are excisions. Consider the diagram

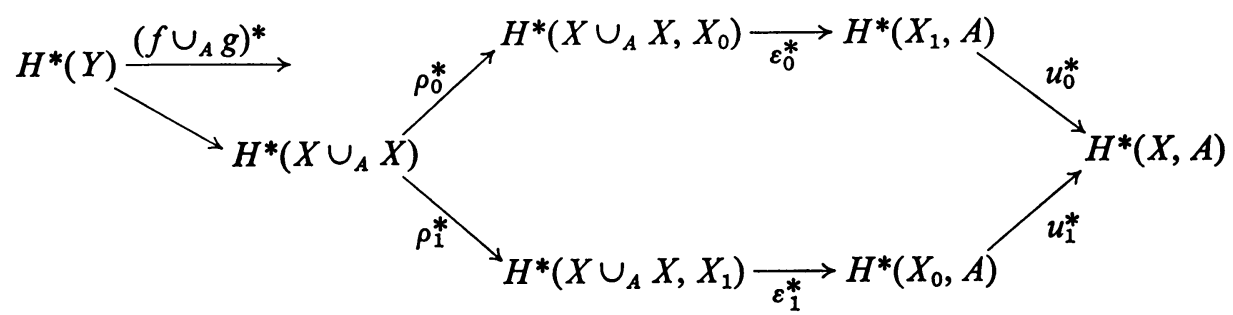

where $u_{i}:(X, A) \rightarrow\left(X_{i}, A\right), i=0,1$, are the folding maps. It is now straightforward to verify that

and so we note

$$
(f-g)^{*}=\left(u_{1}^{*} \varepsilon_{1}^{*} \rho_{1}^{*}-u_{0}^{*} \varepsilon_{0}^{*} \rho_{0}^{*}\right)\left(f \cup_{A} g\right)^{*}
$$

Proposition 4.1 (Steenrod). If $p$ is a prime then the map

$$
(f-g)^{*}: H^{*}\left(Y ; Z_{p}\right) \rightarrow H^{*}\left(X, A ; Z_{p}\right)
$$

commutes with the Steenrod reduced pth powers and the Bockstein coboundary. 
We turn now to a description of $F^{0} H^{*}\left(X \times_{B} Y\right)$ and $F^{-1} H^{*}\left(X \times_{B} Y\right)$. So suppose given a diagram

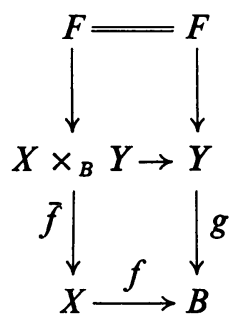

as in $\S 3$. We will denote its Eilenberg-Moore spectral sequence by $\left\{E_{r}, d_{r}\right\}$. $\left\{F^{-p} H^{*}\left(X \times_{B} Y\right)\right\}$ will denote the associated filtration of $H^{*}\left(X \times_{B} Y\right)$.

Proposition 4.2. $F^{0} H^{*}\left(X \times_{B} Y\right)=\operatorname{im}\left\{H^{*}(X) \otimes H^{*}(Y) \rightarrow H^{*}\left(X \times_{B} Y\right)\right\}$.

Proof. Follows directly from Proposition 1.4.

Recall that $X \times_{B} Y \subset X \times Y$ and we have defined maps

$$
\begin{aligned}
& \overline{\bar{f}}: X \times Y \rightarrow X \times B \times Y \text { by } \overline{\bar{f}}(x, y)=(x, f(x), y), \\
& \overline{\bar{g}}: X \times Y \rightarrow X \times B \times Y \text { by } \overline{\bar{g}}(x, y)=(x, g(y), y),
\end{aligned}
$$

note that

$$
\overline{\bar{f}}\left|X \times_{B} Y=\overline{\bar{g}}\right| X \times_{B} Y
$$

and thus we have defined a difference homomorphism

$$
H^{*}(X \times B \times Y) \stackrel{(\overline{\bar{f}}-\overline{\bar{g}})^{*}}{\longrightarrow} H^{*}\left(X \times Y, X \times_{B} Y\right) .
$$

Let

$$
\delta: H^{*}\left(X \times_{B} Y\right) \rightarrow H^{*}\left(X \times Y, X \times_{B} Y\right)
$$

be the coboundary operator of the pair $\left(X \times Y, X \times_{B} Y\right)$.

We now define an additive relation

$$
\Phi: H^{*}(X) \otimes \overline{H^{*}(B)} \otimes H^{*}(Y) \rightarrow H^{*}\left(X \times_{B} Y\right)
$$

by the composition

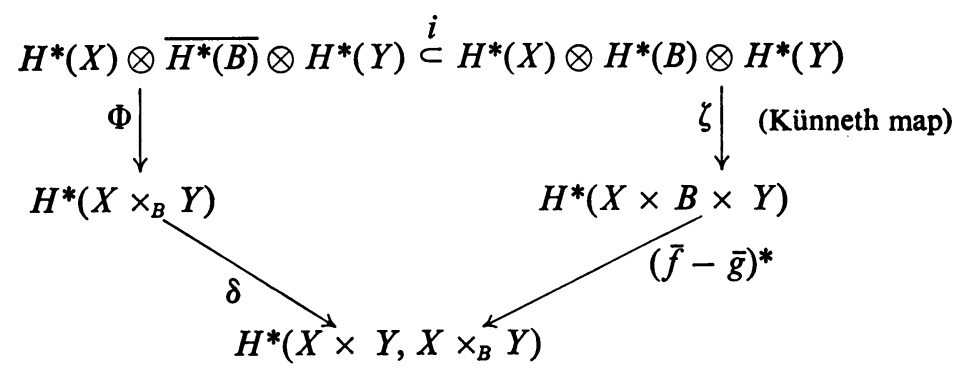

THEOREM 4.3. $F^{-1} H^{*}\left(X \times_{B} Y\right)=\left\{w \in H^{*}\left(X \times_{B} Y\right) \mid \exists z\right.$ with $\left.\Phi(z)=w\right\}$ for some $z \in H^{*}(X) \otimes \overline{H^{*}(B)} \otimes H^{*}(Y)$. 
Proof. Since $B$ is simply connected we can base our calculation of the cohomology of $B$ on singular simplices which have their one-dimensional skeleton at the basepoint. The results of $\$ 3$ can be carried out for this cochain complex in place of $C^{*}(B)$. We shall assume this done and let $C^{*}(B)$ now stand for the cochains based on simplices with their one-dimensional skeleton at the basepoint. We note then that $C^{*}(B)$ is a simply connected algebra in the sense of $\$ 2$. We can therefore use Proposition 2.7 to describe $F^{-1} \operatorname{Tor}_{C^{*}(B)}\left(C^{*}(X), C^{*}(Y)\right)$; we will continue to employ the notation of that result.

Now $F^{-1} H^{*}\left(X \times_{B} Y\right)$ is just the image of $F^{-1} \operatorname{Tor}_{C^{*}(B)}\left(C^{*}(X), C^{*}(Y)\right)$ under the map $\theta$ of Proposition 3.2.

Using these results the proof of Theorem 4.3 reduces to showing that the diagram

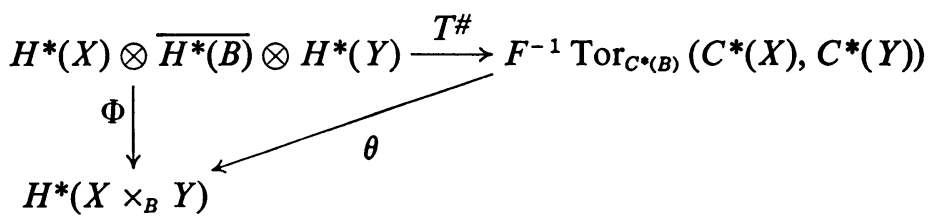

is commutative. Here of course $\Phi$ and $T^{\#}$ are additive relations. The demonstration of commutativity will be by direct calculation.

Let $\phi: H^{*}(X) \otimes \overline{H^{*}(B)} \otimes H^{*}(Y) \rightarrow H^{*}(X) \otimes H^{*}(Y)$ be as in Proposition 2.7. We begin by showing that

$$
v \in \operatorname{ker} \phi \quad \text { iff }(\overline{\bar{f}}-\overline{\bar{g}}) * \zeta i(v) \in \operatorname{im} \delta .
$$

To this end consider the diagram

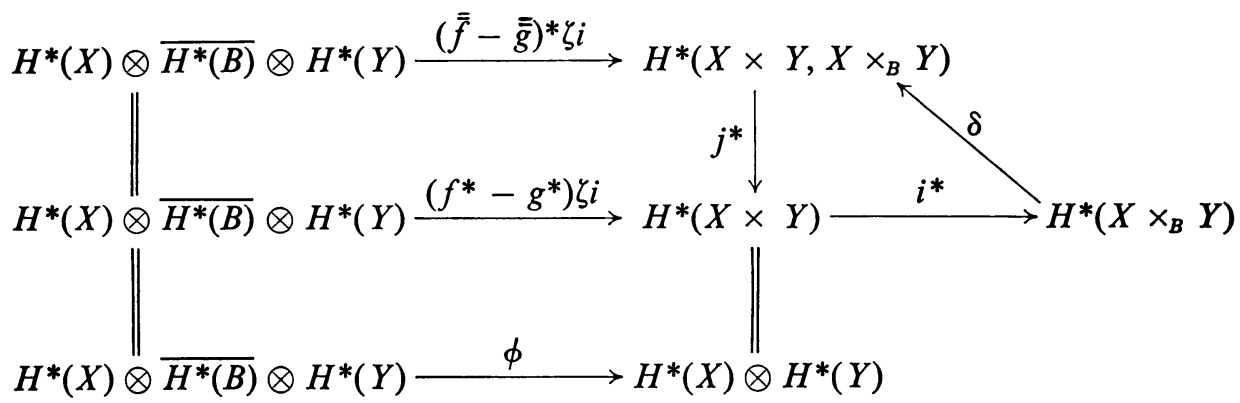

where the squares commute, the triangle is exact and the vertical identification on the right is a Künneth identification. We then have

$$
\begin{aligned}
(\overline{\bar{f}}-\overline{\bar{g}})^{*} \zeta i(v) \in \operatorname{im} \delta & \text { iff } j^{*}(\overline{\bar{f}}-\overline{\bar{g}})^{*} \zeta i(v)=0, \\
& \text { iff }\left(\overline{\bar{f}}^{*}-\overline{\bar{g}}^{*}\right) \zeta i(v)=0, \\
& \text { iff } \phi(v)=0,
\end{aligned}
$$

and therefore $\Phi$ and $T^{\#}$ have the same domain, i.e. they are defined on the same classes. 
It is immediate from the definitions that the indeterminacy of $\phi$ is $\operatorname{ker} \delta=\operatorname{im} i^{*}$ $=F^{0} H^{*}\left(X \times_{B} Y\right)$ and so both $\Phi$ and $T^{\#}$ have the same indeterminacy.

Let us suppose that $v=\sum x_{i} \otimes a_{i} \otimes y_{i} \in \operatorname{ker} \phi$ and that $\bar{x}_{i}, \bar{a}_{i}, \bar{y}_{i}$ are representative cocycles for $x_{i}, a_{i}, y_{i}$ respectively. Since $v \in \operatorname{ker} \phi$ there exists

$$
U \in C^{*}(X) \otimes C^{*}(Y)
$$

such that

$$
d(U)=\sum \bar{x}_{i} \bar{a}_{i} \otimes \bar{y}_{i}-\bar{x}_{i} \otimes \bar{a}_{i} \bar{y}_{i}
$$

using the definition of $T^{\#}$ and $\theta$ we see that

$$
\theta T^{\#}(v)=\operatorname{cls}(\alpha(U)) \in H^{*}\left(X \times_{B} Y\right)
$$

where $\alpha$ was defined in Lemma 3.1.

We now compute $\Phi(v)$. To this end consider the diagram

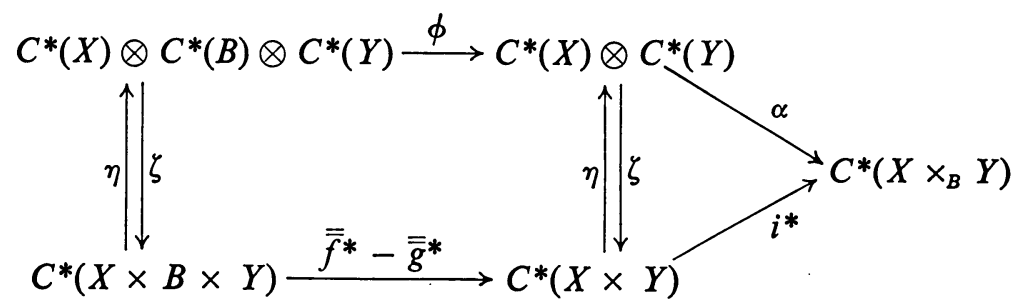

where $\eta$ and $\zeta$ are the usual Künneth chain equivalences. While the above square does not commute, it does commute when we pass to homology.

By definition

$$
\begin{aligned}
\Phi(v) & =\operatorname{cls}\left\{i^{*} d^{-1}\left(\overline{\bar{f}}^{*}-\bar{g}^{*}\right) \zeta\left(\sum \bar{x}_{i} \otimes \bar{a}_{i} \otimes \bar{y}_{i}\right)\right\}, \\
& =\operatorname{cls}\left\{i^{*} d^{-1}\left(\sum \bar{x}_{i} \bar{a}_{i} \otimes \bar{b}_{i}-\bar{x}_{i} \otimes \bar{a}_{i} \bar{y}_{i}\right)\right\}, \\
& =\operatorname{cls}\{i * \zeta(U)\}
\end{aligned}
$$

( $d=$ differential on $C^{*}(X) \otimes C^{*}(Y)$ ), and since the diagram commutes when we pass to homology we have

$$
\Phi(v)=\operatorname{cls}\left(i^{*} \zeta(U)\right)=\operatorname{cls} \alpha(U)=\theta T^{\#}(v),
$$

which completes the proof.

Corollary 4.4. If $k=Z_{p}$, $p$ a prime, then $F^{-1} H^{*}\left(X \times_{B} Y\right)$ is closed under the action of the Steenrod algebra $\mathscr{A}(p)$ on $H^{*}\left(X \times_{B} Y\right)$, i.e. $F^{-1} H^{*}\left(X \times_{B} Y\right)$ is an $\mathscr{A}(p)$ submodule of $H^{*}\left(X \times_{B} Y\right)$.

CONJECTURE. If $k=Z_{p}, p$ a prime, then $F^{-n} H^{*}\left(X \times_{B} Y\right)$ is an $\mathscr{A}(p)$ submodule of $H^{*}\left(X \times_{B} Y\right)$.

In [17] we used a slightly different but equivalent description of ${ }_{1} H^{*}\left(X \times_{B} Y\right)$. The details of the equivalence are left to the reader.

The additive relation $\Phi$ can be given a more familiar interpretation in a case of special interest. We proceed to describe this now. 
Proposition 4.5. If $X=$ point and $Y=P B$, the path space on $B$, then $\Phi=\sigma^{*}$, the cohomology suspension.

Proof. Let us recall that the cohomology suspension is defined by

$$
H^{*}(B, *) \stackrel{g^{*}}{\longrightarrow} H^{*}(P B, \Omega B) \stackrel{\delta}{\longleftarrow} H^{*}(\Omega B)
$$

whereas $\Phi$ is defined by

$$
\overline{H^{*}(B)}=H^{*}(*) \otimes \overline{H^{*}(B)} \otimes H^{*}(P B) \stackrel{(\overline{\bar{f}}-\overline{\bar{g}})^{*}}{\longrightarrow} H^{*}(P B, \Omega B)
$$

It is now a routine matter to verify that

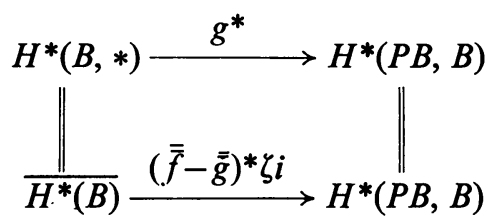

is a commutative diagram (return to the cochain level and use the definition (*)). From this the result follows.

WARNING. If $X=$ point but $Y \neq P B$ then it is in general false that

$$
F^{-1} H^{*}(F)=\operatorname{im} \sigma^{*} \text {. }
$$

COROllary 4.6. If we identify $H^{*}(\Omega B)$ and $\operatorname{Tor}_{C^{*}(B)}(k, k)$ then the cohomology suspension is given by

$$
\begin{gathered}
\sigma^{*}(x)=\operatorname{cls}[z] \in \underset{\|}{H\left[k \otimes_{C^{*}(B)} B\left(C^{*}(B), k\right)\right]} \\
\operatorname{Tor}_{C^{*}(B)}(k, k)
\end{gathered}
$$

where $z \in C^{*}(B)$ is a cocycle representing $x$.

ACKNOWLEDGement. Corollary 4.6 was suggested by the work of Moore in [13].

Using the description of the cohomology suspension given in Corollary 4.6 we can readily establish

Corollary 4.7 (G. W. WhiteheAd). If $B$ is a $q$-connected space, $q \geqq 1$, then the cohomology suspension

$$
s^{*}: Q H^{*}(B)^{n+1} \rightarrow P H^{*}(\Omega B)^{n}
$$

is a monomorphism for $n \leqq 3 q$.

Proof. We can identify $Q H^{*}(B)$ with $\operatorname{Tor}_{H^{*}(B)}^{-1 *}(k, k)=E_{2}^{-1, *}$ as graded vector spaces. Hence using Corollary 4.6 it suffices to show that no element of $E_{2}^{-1, n+1}$, $n \leqq 3 q$, is a $d_{r}$ boundary for any $r \geqq 2$. 
To this end note that $H^{n}(B)=0$ for $0<n \leqq q$. So using the bar construction we readily see that

Recall that

$$
E_{2}^{-t, n}=\operatorname{Tor}_{H}^{-t, n}(k)(k, k)=0 \text { for } n \leqq t q .
$$

and so we see that

$$
d_{t-1}: E_{2}^{-t, n} \rightarrow E_{2}^{-1, n+t-2},
$$

$$
\operatorname{im}\left\{d_{t-1}: E_{2}^{-t, n} \rightarrow E_{2}^{-1, n+t-2}\right\}=0 \text { if } n \leqq t q ;
$$

since

and

$$
n+t-2 \leqq t q+t-2 \text { iff } n \leqq t q
$$

$$
t q+t-2 \geqq 3 q+1
$$

since $t>2$ ), it follows that no element of $E_{2}^{-1, s+1}$, where $s \leqq 3 q$, is in the image of any $d_{r}, r \geqq 2$.

Part II. Commutative Algebra, Collapse Theorems, Applications

In this chapter we will develop some techniques to facilitate the computation of $\operatorname{Tor}_{\Lambda}(A, B)$ when $\Lambda, A$ and $B$ are commutative algebras. This will enable us to compute the $E_{2}$ term of the Eilenberg-Moore spectral sequence in many cases of geometric interest. We obtain simple conditions which imply that $E_{2}=E_{\infty}$ and give applications at the end.

1. The Koszul complex and ESP-sequences. In this section we shall review some very old algebraic ideas in modern guise. As usual the ground ring will be a field $k$ and all modules in sight will be of finite type.

Let $\Lambda$ be a graded commutative connected algebra over $k$. A sequence of elements $\lambda_{1}, \ldots, \lambda_{n}, \ldots$ is called an ESP-sequence in $\Lambda$ iff $\lambda_{i+1}$ is not a zero divisor in the quotient algebra $\Lambda /\left(\lambda_{1}, \ldots, \lambda_{i}\right)$ for each $i$. Here $\left(\lambda_{1}, \ldots, \lambda_{i}\right)$ denotes the ideal generated by $\lambda_{1}, \ldots, \lambda_{i}$ in $\Lambda$. An ideal $I \subset \Lambda$ is called a Borel ideal if there is an ESP-sequence $\lambda_{1}, \ldots, \lambda_{n}, \ldots$ in $\Lambda$ with $\operatorname{deg} \lambda_{i}>0$ and $I=\left(\lambda_{1}, \ldots, \lambda_{n}, \ldots\right)$.

Let $x_{1}, \ldots, x_{n}, \ldots$ be a sequence of elements of $\Lambda$. By $E\left[u_{1}, \ldots, u_{n}, \ldots\right]$ denote an exterior algebra over $k$ on generators $u_{1}, \ldots, u_{n}, \ldots$ The Koszul complex of $\Lambda$ relative to the elements $x_{1}, \ldots, x_{n}, \ldots \in \Lambda$ is defined to be the complex

the boundary is given by

$$
\mathscr{E}=\Lambda \otimes E\left[u_{1}, \ldots, u_{n}, \ldots\right]
$$

$$
\begin{aligned}
& d\left(\lambda \otimes u_{i}\right)=\lambda x_{i} \otimes 1, \\
& d(\lambda \otimes 1)=0,
\end{aligned}
$$

and requiring that $d$ be a derivation of algebras. We give $\mathscr{E}$ a graded structure by setting

$$
\begin{aligned}
\text { bideg } \lambda & =(0, \operatorname{deg} \lambda), \quad \text { all } \lambda \in \Lambda, \\
\text { bideg } u_{i} & =\left(-1, \operatorname{deg} x_{i}\right), \quad i=1, \ldots,
\end{aligned}
$$

clearly $d^{2}=0$ and so $\mathscr{E}$ is a differential algebra. One easily checks that

$$
H^{0}(\mathscr{E})=\Lambda /\left(x_{1}, \ldots, x_{n}, \ldots\right) \text {. }
$$


If $x_{1}, \ldots, x_{n}, \ldots \in \Lambda$ we denote by $P\left[\bar{x}_{1}, \ldots, \bar{x}_{n}, \ldots\right]$ a polynomial algebra on generators $\bar{x}_{1}, \ldots$ of degree deg $x_{i}$. (If the characteristic of $k$ is not 2 we assume that $\operatorname{deg} x_{i}$ is even.) Define $\phi: P\left[\bar{x}_{1}, \ldots, \bar{x}_{n}, \ldots\right] \rightarrow \Lambda$ by $\phi\left(\bar{x}_{i}\right)=x_{i}$. This gives a $P\left[\bar{x}_{1}, \ldots, \bar{x}_{n}, \ldots\right]$-module structure on $\Lambda$.

We shall need the following general result concerning ESP-sequences.

Proposition 1.1. The following conditions are equivalent:

(a) $x_{1}, \ldots, x_{n}, \ldots \in \Lambda$ is an ESP-sequence;

(b) the Koszul complex $\mathscr{E}$ is acyclic;

(c) $\Lambda$ is a free $P\left[\bar{x}_{1}, \ldots, \bar{x}_{n}, \ldots\right]$-module.

The equivalence of (a) and (b) follows by a slight extension from the finite case of the proofs in [11, §7], and so it suffices to prove the equivalence of (b) and (c). However, before turning to this we record an immediate consequence of the equivalence of (a) and (b).

Corollary 1.2. Let $\Gamma=P\left[y_{1}, \ldots, y_{n}, \ldots\right]$ and suppose that $A$ is any $\Gamma$-module, then

$$
\begin{aligned}
\operatorname{Tor}_{\Gamma}(A, k) & =H\left[A \otimes E\left[u_{1}, \ldots, u_{n}, \ldots\right], d\right], \\
d\left(a \otimes u_{i}\right) & =a y_{i} \otimes 1 .
\end{aligned}
$$

Proof. Note that $y_{1}, \ldots, y_{n}, \ldots$ is an ESP-sequence in $\Gamma$. It therefore follows from (a) $\Rightarrow$ (b) that $\mathscr{E}$ is a $\Gamma$ free resolution of $k$, and the result follows.

Proof of $(b) \Leftrightarrow(c)$. Suppose that the Koszul complex $\mathscr{E}$ is acyclic. Then by Corollary 1.2 we have

$$
\operatorname{Tor}_{P\left[x_{1}, \ldots\right]}^{-1, *}(\Lambda, k)=H(\mathscr{E})=0 .
$$

Therefore by [5, Theorem 6.1, p. 156] $\Lambda$ is a free $P\left[\bar{x}_{1}, \ldots\right]$-module. If $\Lambda$ is a free $P\left[\bar{x}_{1}, \ldots\right]$-module then we have

$$
0=\operatorname{Tor}_{P\left[x_{1}, \ldots\right]}^{-n, *}(\Lambda, k)=H(\mathscr{E}), \quad n<0,
$$

and the result follows.

Recall that $x_{1}, \ldots, x_{n}, \ldots \in \Lambda$ are called algebraically independent if the map

is a monomorphism.

$$
\phi: P\left[\bar{x}_{1}, \ldots, \bar{x}_{n}, \ldots\right] \rightarrow \Lambda
$$

CoRollary 1.3. If $x_{1}, \ldots, x_{n}, \ldots \in \Lambda$ is an ESP-sequence then $x_{1}, \ldots, x_{n}, \ldots$ are algebraically independent.

Corollary 1.4. If $x_{1}, \ldots, x_{n}, \ldots \in \Lambda$ is an ESP-sequence then the subalgebra generated by $x_{1}, \ldots, x_{n}, \ldots$ is isomorphic to $P\left[\bar{x}_{1}, \ldots, \bar{x}_{n}, \ldots\right]$.

Note that $x y, y z$ are algebraically independent in the polynomial algebra $P[x, y, z]$ but are not an ESP-sequence.

2. Some computations. Suppose that $\Lambda$ is a graded commutative connected algebra over $k$ and $I \subset \Lambda$ is a Borel ideal. How could we go about constructing an ESP-sequence generating $I$ ? In a graded algebra there is a fairly natural way to 
choose a minimal set of generators for the ideal $I$. This is done as follows: Since $I$ inherits a graded structure from $\Lambda$ we can look in the lowest degree in which $I$ is nonzero and there choose a vector space basis, say $x_{1}, \ldots, x_{p}$. Now if

$$
I=\left(x_{1}, \ldots, x_{p}\right)
$$

we have constructed a minimal ideal basis for $I$. If $I \neq\left(x_{1}, \ldots, x_{p}\right)$ then set $I_{1}=\left(x_{1}, \ldots, x_{p}\right)$ and consider the first degree in which $I \neq I_{1}$. Then in this degree we can choose a direct sum decomposition $I=I_{1} \oplus \hat{I}_{1}$. Choose a vector space basis for $I_{1}$, and denote it by $x_{p+1}, \ldots, x_{q}$. Set $I_{2}=\left(x_{1}, \ldots, x_{q}\right)$. If $I=I_{2}$ we are done, if not just continue to repeat the above process until we arrive at an ideal basis for $I$. The base that we arrive at is minimal in the sense that $I \neq\left(x_{1}, \ldots, x_{s}, \ldots\right)$ for any $s$. Further, all minimal ideal bases can be obtained for $I$ by the above process.

We record now some elementary lemmas.

LemMA 2.1. Let $X=\left(x_{1}, \ldots, x_{n}, \ldots\right)$ be a minimal ideal basis for an ideal $I \subset \Lambda$ arranged in order of increasing degrees. Suppose that $x_{1}, \ldots, x_{r}$ are the elements of minimal degree. Then $x_{1}, \ldots, x_{r}$ are a vector space basis for $I$ in the minimal degree for which $I \neq 0$.

Lemma 2.2. With the notation of Lemma 2.1 let $J=\left(x_{1}, \ldots, x_{r}\right) \subset \Lambda$. Then the collection of equivalence classes $X^{\prime}=\left(\left[x_{r+1}\right], \ldots,\left[x_{n}\right], \ldots\right)$ is a minimal ideal basis for the ideal $I / J \subset \Lambda / J$.

LEMMA 2.3. If $I \subset \Lambda$ is a Borel ideal and $\lambda_{1}, \ldots, \lambda_{n}, \ldots$ is an ESP-sequence generating $I$, then $\lambda_{1}, \ldots, \lambda_{n}, \ldots$ is a minimal ideal basis for $I$.

LEMMA 2.4. Suppose that $V \subset \Lambda$ is a vector subspace and that $X=\left\{x_{i}\right\}$ and $Y=\left\{y_{j}\right\}$ are vector space bases for $V$. Then $x_{1}, \ldots$ is an ESP-sequence if and only if $y_{i}, \ldots$ is an ESP.

Proof. For each $i$ we have elements $a_{i j} \in k, j=1, \ldots, n(i)$, so that $\sum a_{i j} x_{j}=y_{i}$. Similarly for each $j$ we have elements $b_{j s} \in k$ with $x_{j}=\sum b_{j s} y_{s}$. We then have

$$
y_{i}=\sum a_{i j} b_{j s} y_{s}, \quad x_{j}=\sum b_{j s} a_{s i} x_{i},
$$

and since the sets $X$ and $Y$ are bases it follows that

$$
\begin{aligned}
& \sum a_{i j} b_{j s}=\delta_{i s}, \\
& \sum b_{j s} a_{s i}=\delta_{j i} .
\end{aligned}
$$

If we now form the Koszul complexes

$$
\begin{aligned}
\mathscr{E}=\Lambda \otimes E\left[u_{1}, \ldots\right], & d\left(u_{i}\right)=x_{i}, \\
\mathscr{E}^{\prime}=\Lambda \otimes E\left[v_{1}, \ldots\right], & d^{\prime}\left(v_{i}\right)=y_{i},
\end{aligned}
$$

we know by Proposition 1.1 that $\mathscr{E}$ is acyclic. We now define maps

$$
T: \mathscr{E} \rightarrow \mathscr{E}^{\prime}, \quad T^{\prime}: \mathscr{E}^{\prime} \rightarrow \mathscr{E}
$$

by

$$
\begin{aligned}
T(\lambda \otimes 1) & =\lambda \otimes 1, & & T^{\prime}(\lambda \otimes 1)=\lambda \otimes 1 \\
T\left(\lambda \otimes u_{i}\right) & =\sum b_{i j} \lambda \otimes v_{j}, & T^{\prime}\left(\lambda \otimes v_{j}\right) & =\sum a_{j s} \lambda \otimes u_{s} .
\end{aligned}
$$


It is then straightforward to verify that $T$ and $T^{\prime}$ are maps of complexes. Furthermore direct calculation shows

$$
T^{\prime} T=1: \mathscr{E} \rightarrow \mathscr{E} \quad \text { and } T T^{\prime}=1: \mathscr{E}^{\prime} \rightarrow \mathscr{E}^{\prime}
$$

and therefore $\mathscr{E}$ and $\mathscr{E}^{\prime}$ are isomorphic as complexes. Since $\mathscr{E}$ is acyclic so is $\mathscr{E}^{\prime}$. Therefore by Proposition $1.1 y_{1}, \ldots$ is an ESP-sequence.

LEMMA 2.5. $\lambda_{1}, \ldots, \lambda_{n}, \ldots$ is an ESP-sequence in $\Lambda$ iff $\lambda_{1}, \ldots, \lambda_{n}$ is an ESPsequence in $\Lambda$ and $\lambda_{n+1}, \ldots$ is an ESP-sequence in $\Lambda /\left(\lambda_{1}, \ldots, \lambda_{n}\right)$.

Proof. First note that from Proposition 1.1 it follows that the property of being an ESP-sequence is independent of the ordering of the elements. The result then follows directly from the definition of an ESP-sequence.

Proposition 2.6. Suppose that $I \subset \Lambda$ is a Borel ideal and that $x_{1}, \ldots$ is a minimal ideal basis for $I$, then $x_{1}, \ldots$ is an ESP-sequence in $\Lambda$.

Proof. Since $I \subset \Lambda$ is a Borel ideal we can choose an ESP-sequence $\lambda_{1}, \ldots, \lambda_{n}, \ldots$ generating $I$. We may assume that the $\lambda_{i}$ are arranged in order of increasing degree. Similarly we may assume that $x_{1}, \ldots, x_{n}, \ldots$ are arranged in order of increasing degree.

Let $x_{1}, \ldots, x_{n}$ and $\lambda_{1}, \ldots, \lambda_{m}$ be the elements of minimal degree. Then using Lemma 2.3 and Lemma 2.1 we see that $x_{1}, \ldots, x_{n}$ and $\lambda_{1}, \ldots, \lambda_{m}$ are vector space bases for the same vector space. Therefore by Lemma $2.4 x_{1}, \ldots, x_{n}$ is an ESPsequence in $\Lambda$.

We can now complete the proof by induction on the degree of $x_{i}$ as follows. Suppose that the elements of degree less than $n, x_{1}, \ldots, x_{r}$, have already been shown to be an ESP-sequence. Let $x_{r+1}, \ldots, x_{s}$ be the elements of degree $n$.

Denote by $\lambda_{1}, \ldots, \lambda_{r^{\prime}}$, the elements of degree $<n$. Repeated application of the above argument and Lemma 2.2 shows that

$$
\Lambda /\left(x_{1}, \ldots, x_{r}\right)=\Lambda /\left(\lambda_{1}, \ldots, \lambda_{r^{\prime}}\right)
$$

and that $\left[x_{r+1}\right], \ldots$ is a minimal ideal basis for

$$
I /\left(x_{1}, \ldots, x_{r}\right) \subset \Lambda /\left(x_{1}, \ldots, x_{r}\right)=\Lambda /\left(\lambda_{1}, \ldots, \lambda_{r^{\prime}}\right) .
$$

If $\lambda_{r^{\prime}+1}, \ldots, \lambda_{s^{\prime}}$ are the elements of degree $n$ we see that

$$
\left[x_{r+1}\right], \ldots,\left[x_{s}\right], \quad\left[\lambda_{r^{\prime}+1}\right], \ldots,\left[\lambda_{s^{\prime}}\right],
$$

are vector space bases for the same vector space, namely the vector space spanned by the elements of lowest degree in $I /\left(x_{1}, \ldots, x_{r}\right)$.

By Lemma $2.5\left[\lambda_{r^{\prime}+1}\right], \ldots,\left[\lambda_{s^{\prime}}\right]$ is an ESP-sequence in

$$
\Lambda /\left(\lambda_{1}, \ldots, \lambda_{r^{\prime}}\right)=\Lambda /\left(x_{1}, \ldots, x_{r}\right) .
$$

Therefore by Lemma $2.4\left[x_{r+1}\right], \ldots,\left[x_{s}\right]$ is an ESP-sequence in $\Lambda /\left(x_{1}, \ldots, x_{r}\right)$ and so by Lemma $2.5 x_{1}, \ldots, x_{r}, x_{r+1}, \ldots, x_{s}$ is an ESP-sequence in $\Lambda$.

This completes the proof by induction on $\operatorname{deg} x_{i}$. 
If $I \subset \Lambda$ is a Borel ideal and $x_{1}, \ldots, x_{n}, \ldots$ is a minimal ideal basis for $I$, then

$$
\mathscr{E}=\Lambda \otimes E\left[u_{1}, \ldots, u_{n}, \ldots\right], \quad d\left(u_{i}\right)=x_{i}
$$

is an acyclic complex and hence provides a small $\Lambda$-free resolution of $\Lambda / I$. Thus we can conclude

Proposition 2.7. If $I \subset \Lambda$ is a Borel ideal, then

$$
\operatorname{Tor}_{\Lambda}(k, \Lambda / I)=E\left[u_{1}, \ldots\right]
$$

as an algebra, where the homological degree of $u_{i}$ is -1 .

We are seeking a generalization of this result for the case where we replace $k$ by some other $\Lambda$-module $B$. To do this we shall employ the following change of rings result.

THEOREM 2.8. Let $\Lambda$ be an algebra and $\Gamma$ a subalgebra. Set $\Omega=\Lambda / / \Gamma$. Suppose that $\Lambda$ is a free $\Gamma$-module and we are given $\left(A_{\Omega},{ }_{\Lambda} C\right)$, then there exists a spectral sequence $\left\{E_{r}, d_{r}\right\}$ with

$$
E_{r} \Rightarrow \operatorname{Tor}_{\Lambda}(A, C), \quad E_{2}^{p, q}=\operatorname{Tor}_{\Omega}^{p}\left(A, \operatorname{Tor}_{\Gamma}^{q}(k, C)\right) .
$$

Proof. See [5, p. 349].

Proposition 2.9. Suppose $P\left[x_{1}, \ldots\right] \rightarrow A$ is a map of algebras with $J=((\mathrm{im}) \bar{f}) \subset A$ a Borel ideal. Then

$$
\operatorname{Tor}_{P\left[x_{1}, \ldots\right]}(A, k)=A / J \otimes E\left[u_{1}, \ldots\right]
$$

as algebras, where deg $u_{i}=(-1, *)$.

Proof. From among the elements $f\left(x_{1}\right), \ldots, f\left(x_{n}\right), \ldots$ select a minimal ideal basis for $J$. Now by rearranging $x_{1} \ldots, x_{n}, \ldots$ we may assume that

$$
P\left[x_{1}, \ldots, x_{n}, \ldots\right]=P\left[y_{1}, \ldots, z_{n}, \ldots\right]
$$

where $f\left(y_{1}\right), \ldots, f\left(y_{m}\right), \ldots$ is a minimal ideal basis for $J$ and hence an ESP-sequence in $A$.

Now let $\Gamma=P\left[y_{1}, \ldots\right]$. Then $P\left[x_{1}, \ldots\right]$ is a free $\Gamma$-module and so by Theorem 2.3 we obtain a spectral sequence

$$
E_{r} \Rightarrow \operatorname{Tor}_{P\left[x_{1}, \ldots\right]}(A, k), \quad E_{2}=\operatorname{Tor}_{\Omega}\left(\operatorname{Tor}_{\Gamma}(A, k), k\right),
$$

where

$$
\Omega=P\left[x_{1}, \ldots\right] / / \Gamma=P\left[z_{1}, \ldots\right] .
$$

Now since $f\left(y_{1}\right), \ldots$ is an ESP-sequence in $A$ we have by Proposition 1.1 that $A$ is a free $\Gamma$-module. Thus

$$
\begin{aligned}
\operatorname{Tor}_{\Gamma}(A, k) & =A \otimes_{\Gamma} k=A / J \\
& \Rightarrow E_{2}^{p, q}=0 \quad q \neq 0 \\
& \Rightarrow \operatorname{Tor}_{P\left[x_{1}, \ldots\right]}(A, k)=\operatorname{Tor}_{P\left[z_{1}, \ldots\right]}(A / J, k) .
\end{aligned}
$$


Now $A / J$ is a trivial $P\left[z_{1}, \ldots\right]$-module and so using a Koszul complex we see

$$
\operatorname{Tor}_{P\left[x_{1}, \ldots\right]}(A, k)=A \mid J \otimes E\left[u_{1}, \ldots\right],
$$

where $\operatorname{deg} u_{i}=\left(-1, \operatorname{deg} z_{i}\right)$.

Proposition 2.10. Suppose given algebras $A, \Lambda, B$ and maps of algebras

$$
A \stackrel{f}{\longleftarrow} \Lambda \stackrel{\phi}{\longrightarrow} B
$$

where

(a) $\phi$ is onto,

(b) $\operatorname{ker} \phi$ is a Borel ideal in $\Lambda$,

(c) $f((\mathrm{ker}) \phi)$ generates a Borel ideal $J \subset A$;

then as algebras

$$
\operatorname{Tor}_{\Lambda}(A, B)=A / J \otimes E\left[u_{1}, \ldots\right]
$$

where the homological degree of $u_{i}$ is -1 .

Proof. Let $\lambda_{1}, \ldots, \lambda_{n}, \ldots$ be an ESP-sequence generating $\operatorname{ker} \phi$ and let $\Gamma$ be the subalgebra of $\Lambda$ generated by $\lambda_{1}, \ldots, \lambda_{n}, \ldots$ Then by Corollary 1.4

$$
\Gamma=P\left[\bar{\lambda}_{1}, \ldots\right]
$$

and by Proposition $1.1 \Lambda$ is a free $\Gamma$ module. Thus by Theorem 2.3 we have a spectral sequence

$$
E_{r} \Rightarrow \operatorname{Tor}_{\Lambda}(A, B), \quad E_{2}=\operatorname{Tor}_{\Lambda / \Gamma}\left(\operatorname{Tor}_{\Gamma}(A, k), B\right)
$$

but $\Lambda / \Gamma=B$ and so

$$
E_{2}^{-p, *}=0(p>0), \quad E_{2}^{0, *}=\operatorname{Tor}_{\Gamma}(A, k),
$$

but by Proposition 2.9

$$
\operatorname{Tor}_{\Gamma}(A, k)=A / J \otimes E\left[u_{1}, \ldots\right]
$$

which completes the proof.

3. Collapse theorems. In this section we shall apply the calculations of the previous section to obtain collapse theorems for the Eilenberg-Moore spectral sequence. Examples will be presented in the next section.

Throughout this section we will be studying a fixed diagram

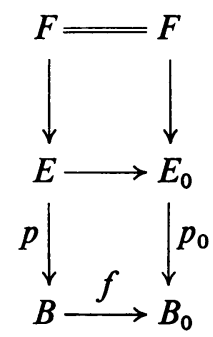


where

(a) $F \rightarrow E_{0} \rightarrow B_{0}$ is a Serre fibre space,

(b) $F \rightarrow E \rightarrow B$ is the induced fibre space by the map $f: B \rightarrow B_{0}$,

(c) $B_{0}$ is simply connected.

We denote by $\left\{E_{r}, d_{r}\right\}$ the Eilenberg-Moore spectral sequence of this diagram. It has

$$
E_{r} \Rightarrow H^{*}(E), \quad E_{2}=\operatorname{Tor}_{H^{*}\left(B_{0}\right)}\left(H^{*}(B), H^{*}\left(E_{0}\right)\right) .
$$

Theorem 3.1 (The Big Collapse Theorem). If

(A) $p_{0}^{*}: H^{*}\left(B_{0}\right) \rightarrow H^{*}\left(E_{0}\right)$ is onto,

(B) $I=\operatorname{ker} p_{0}^{*} \subset H^{*}\left(B_{0}\right)$ is a Borel ideal,

(C) $f^{*}(I)$ generates a Borel ideal $J \subset H^{*}(B)$,

then

(a) $E_{2}=E_{\infty}$,

(b) $E_{2}=H^{*}(B) / J \otimes E\left[u_{1}, \ldots\right]$ where the homological degree of $u_{i}$ is -1 .

Proof. We merely note that (b) follows from Proposition 2.9 and observe that

$$
d_{r}: E_{2}^{-p, *} \rightarrow E_{2}^{-p+r, *}=0 \text { if } p \leqq 1 \text { and } r \geqq 2 ;
$$

but by (b) $E_{2}$ is generated as an algebra by $E_{2}^{0, *}$ and $E_{2}^{-1, *}$, and since $d_{r}$ is a derivation we deduce $E_{2}=E_{\infty}$.

If $B=$ point then $E=F$ and $E_{r} \Rightarrow H^{*}(F)$

Corollary 3.2 (Little Collapse Theorem). If $F \rightarrow E_{0} \rightarrow B_{0}$ is a Serre fibre space over the simply connected space $B_{0}$ and

(A) $p_{0}^{*}: H^{*}\left(B_{0}\right) \rightarrow H^{*}\left(E_{0}\right)$ is onto,

(B) $\operatorname{ker} p_{0}^{*}$ is a Borel ideal in $H^{*}\left(B_{0}\right)$, then

(a) $E_{2}=E_{\infty}$,

(b) $E_{2}=E\left[u_{1}, \ldots\right]$, where the homological degree of $u_{i}$ is -1 .

COROLlary 3.3. Same hypothesis as Theorem 3.1 and in addition assume that characteristic $k \neq 2$; then

$$
H^{*}(E) \cong \operatorname{Tor}_{H^{*}\left(B_{0}\right)}\left(H^{*}(B), H^{*}\left(E_{0}\right)\right)
$$

as algebras.

Proof. By Theorem 3.1 we have a filtration $\left\{F^{-n} H^{*}(E)\right\}$ with

$$
\begin{aligned}
E^{0} H^{*}(E) & =\operatorname{Tor}_{H^{*}\left(B_{0}\right)}\left(H^{*}(B), H^{*}\left(E_{0}\right)\right) \\
& =H^{*}(B) / J \otimes E\left[u_{1}, \ldots\right] .
\end{aligned}
$$

Since the characteristic of $k$ is not 2 this is a free commutative algebra over $H^{*}(B)$ and hence the extension as algebras is trivial. Note that the isomorphism is not natural. 
COROLlary 3.4. Same hypothesis as Corollary 3.2 and in addition assume that the characteristic of $k$ is not 2 ; then

$$
H^{*}(F)=\operatorname{Tor}_{H^{*}\left(B_{0}\right)}\left(k, H^{*}\left(E_{0}\right)\right)
$$

as algebras.

Corollary 3.5. Same hypothesis as Theorem 3.1; then

$$
\operatorname{ker} p^{*}=J \text {. }
$$

Proof. First we know

$$
E_{2}^{0, *}=H^{*}(B) \otimes_{H^{*}\left(B_{0}\right)} H^{*}\left(E_{0}\right)=H^{*}(B) / J .
$$

Next from Proposition 4.2 we have

$$
\begin{aligned}
F^{0} H^{*}(E) & =\operatorname{im}\left\{H^{*}(B) \otimes H^{*}\left(E_{0}\right) \longrightarrow H^{*}(E)\right\} \\
& =\operatorname{im}\left\{H^{*}(B) \otimes_{H^{*}\left(B_{0}\right)} H^{*}\left(E_{0}\right) \longrightarrow H^{*}(E)\right\} \\
& =\operatorname{im}\left\{H^{*}(B) / J \stackrel{p^{*}}{\longrightarrow} H^{*}(E)\right\} .
\end{aligned}
$$

But by Theorem 3.1

$$
F^{0} H^{*}(E) \cong H^{*}(B) / J
$$

and therefore

$$
p^{*}: H^{*}(B) / J \rightarrow H^{*}(E)
$$

is a monomorphism. Hence $J=\operatorname{ker} p^{*}$.

We now turn to results with $Z_{2}=k$ as coefficients. We shall assume that the reader is familiar with the early parts of [11] and [18].

Corollary 3.6 (MASSEY-Peterson). Same as hypothesis Theorem 3.1 and suppose that $k=Z_{2}$; then as $R-\mathscr{A}(2)$ algebras

$$
H^{*}(E)=U_{R}\left(F^{-1}\right),
$$

where

$$
R=H^{*}(B) / \operatorname{ker} p^{*}, \quad F^{-1}=F^{-1} H^{*}(E) .
$$

This isomorphism is natural.

Proof. We remind the reader that $U_{R}$ is defined in [11].

By Theorem 3.1 there is a filtration $\left\{F^{-n} H^{*}(E)\right\}$ with $E^{0} H^{*}(E)=E_{R}\left[u_{1}, \ldots\right]$. The filtration degree of $u_{i}$ being -1 .

By Corollary $3.4 F^{-1}$ is an $\mathscr{A}(2)$ submodule of $H^{*}(E)$, and hence is an $R-\mathscr{A}(2)$ module. Thus the inclusion mapping $F^{-1} \subset H^{*}(E)$ induces a map of $R-\mathscr{A}(2)$ algebras

$$
\zeta: U_{R}\left(F^{-1}\right) \rightarrow H^{*}(E) .
$$

We claim that $\zeta$ is an isomorphism. To see this choose $x_{i} \in H^{*}(E)$ corresponding to $u_{i} \in E^{0} H^{*}(E)$ and write $\bar{x}_{i}$ for $x_{i}$ as an element of $U_{R}\left(F^{-1}\right)$. Note that $\zeta\left(\bar{x}_{i}\right)=x_{i}$. 
The elements $\bar{x}_{1}, \ldots$ form an $R$-module generating set for $F^{-1}$ and hence the monomials in $\bar{x}_{1}, \ldots$ with exponent 0,1 generate $U_{R}\left(F^{-1}\right)$ as an $R$-module. Since the monomials of exponent 0,1 in $u_{1}, \ldots$ are an $R$-basis for $E^{0} H^{*}(E)$ it follows that the monomials in $x_{1}, \ldots$ of exponent 0,1 are an $R$-basis for $H^{*}(E)$.

Now $\zeta$ is a map of algebras and hence maps the monomials in $\bar{x}_{1}, \ldots$ of exponent 0,1 in a one-one-onto fashion to the monomials in $x_{1}, \ldots$ of exponent 0,1 . Thus $\zeta$ maps an $R$-generating set in a one-one-onto fashion to an $R$-basis. Therefore $\zeta$ must be an isomorphism.

Acknowledgement. Professor W. S. Massey suggested that we could obtain a proof of Corollary 3.6 by using the Eilenberg-Moore spectral sequence.

COROLlary 3.7. Same hypothesis as Corollary 3.2 and in addition assume that $k=Z_{2}$. Then

$$
\begin{aligned}
H^{*}(F) & =U_{Z_{2}}\left(F^{-1}\right) \\
& =U(X) \quad\left\{X=x € F^{-1} \mid \operatorname{deg} x>0\right\}
\end{aligned}
$$

as $\mathscr{A}(2)$ algebras.

\section{Examples.}

EXAmPle 1. Homogeneous spaces. Suppose that $G$ is a compact, connected Lie group and $U \subset G$ is a closed connected subgroup. It is very often the case ([3], [4]) that $H^{*}\left(B_{G}\right)$ and $H^{*}\left(B_{U}\right)$ are polynomial algebras and we shall assume that this is so.

Lemma 4.1. Suppose that $\Lambda$ and $\Gamma$ are polynomial algebras and that $f: \Lambda \rightarrow \Gamma$ is an epimorphism of algebras, then

$$
\operatorname{Tor}_{\Lambda}(k, \Gamma)=E\left[u_{1}, \ldots\right]
$$

where the homological degree of $u_{i}$ is -1 .

Proof. Set $A=\Gamma$ and $J=\bar{\Gamma}$ and apply Proposition 2.9.

Consider now the universal bundle

$$
G / U \longrightarrow B_{U} \stackrel{\rho}{\longrightarrow} B_{G} .
$$

Corollary 4.2. Assume that $\rho^{*}: H^{*}\left(B_{G}\right) \rightarrow H^{*}\left(B_{U}\right)$ is onto; then

(a) $\operatorname{char}(k) \neq 2$, then $H^{*}(G / U)=\operatorname{Tor}_{H^{*}\left(B_{G}\right)}\left(k, H^{*}\left(B_{U}\right)\right)$ as an algebra;

(b) $k=Z_{2}$, then $H^{*}(G / U)=U(X)$,

$$
X=\left\{x \in F^{-1} \mid \operatorname{deg} x>0\right\}
$$

as $\mathscr{A}(2)$ algebras.

Since $\rho^{*}: H^{*}\left(B_{G}\right) \rightarrow H^{*}\left(B_{U}\right)$ is onto it is fairly easy, using the material of Part I, to show that $X$ consists entirely of transgressive elements.

EXAMPLE 2. Bundles that are totally $\nsim 0$. Suppose that $\xi_{0}=\left(E_{0}, p_{0}, B_{0}, F\right)$ is a Serre fibre space over the simply connected space $B_{0}$.

Proposition 4.3. $\xi_{0}$ is totally $\nsim 0 \Leftrightarrow H^{*}\left(E_{0}\right)$ is a free $H^{*}\left(B_{0}\right)$ module. 
Proof. $\Rightarrow$ : The Serre spectral sequence of $\xi_{0}$ collapses and there is a filtration on $H^{*}\left(E_{0}\right)$ so that

$$
E^{0}\left(H^{*}\left(E_{0}\right)\right)=H^{*}\left(B_{0}\right) \otimes H^{*}(F) .
$$

But this is a free $H^{*}\left(B_{0}\right)$ module and hence so is $H^{*}\left(E_{0}\right)$.

$\Leftarrow$ : Consider the Eilenberg-Moore spectral sequence of $\xi_{0}$. It has

$$
\begin{aligned}
E_{r} & \Rightarrow H^{*}(F), \\
E_{2} & =\operatorname{Tor}_{H^{*}\left(B_{0}\right)}\left(k, H^{*}\left(E_{0}\right)\right) \\
& =k \otimes_{H^{*}\left(B_{0}\right)} H^{*}\left(E_{0}\right),
\end{aligned}
$$

and hence $E_{2}=E_{\infty}$ and the natural map

$$
k \otimes_{H^{*}\left(B_{0}\right)} H^{*}\left(E_{0}\right) \rightarrow H^{*}(F)
$$

is an isomorphism. Thus

$$
i^{*}: H^{*}\left(E_{0}\right) \rightarrow H^{*}(F)
$$

is onto, and hence $\xi_{0}$ is totally $\nsim 0$.

Suppose that we have a diagram

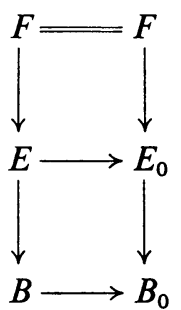

the fibration $\xi=(E, p, B, F)$ being induced from the fibration $\xi_{0}$ by the map $B \rightarrow B_{0}$.

COROLlaRY 4.4. If $\xi_{0}$ is totally $\approx 0$ then

(a) $H^{*}(E)=H^{*}(B) \otimes_{H^{*}\left(B_{0}\right)} H^{*}\left(E_{0}\right)$ as an algebra;

(b) $\xi$ is totally $\approx 0$.

Proof. Obvious from the Eilenberg-Moore spectral sequence of the above diagram.

EXAMPLE 3. Bundles with fibre a Stiefel manifold. Let us consider fibre bundles $\xi=\left(E, p, B, V_{n, r}, S O(n)\right)$ where $V_{n, r}=S O(n) / S O(n-r)$. The universal example for such bundles is $\xi_{0}=\left(B_{S O(n-r)}, \rho, B_{S O(n)}, V_{n, r}, S O(n)\right)$. If we work with $Z_{2}$ coefficients throughout then we have

$$
\begin{aligned}
H^{*}\left(B_{\text {SO }(n)}\right) & =P\left[w_{2}, \ldots, w_{n}\right], \\
H^{*}\left(B_{\text {SO }(n-r)}\right) & =P\left[w_{2}, \ldots, w_{n-r}\right],
\end{aligned}
$$

and $\rho^{*}: H^{*}\left(B_{\text {SO }(n)}\right) \rightarrow H^{*}\left(B_{\text {SO }(n-r)}\right)$ is given by

$$
\begin{aligned}
\rho^{*}\left(w_{i}\right) & =w_{i}, & & 2 \leqq i \leqq n-r, \\
& =0, & & n-r+1 \leqq i \leqq n .
\end{aligned}
$$


Let

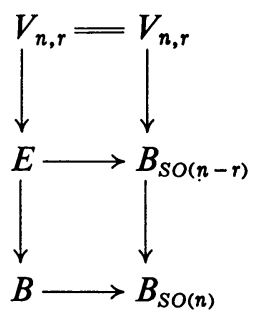

be a classifying diagram for $\xi$. Denote Eilenberg-Moore spectral sequence by $\left\{E_{r}, d_{r}\right\}$.

Proposition 4.5. $\xi$ is totally $\nsim 0 \Leftrightarrow w_{n-r+1}(\xi)=\cdots=w_{n}(\xi)=0$.

Proof. From our calculations in the second section we know that

$$
\begin{aligned}
E_{2} & =H\left[H^{*}(B) \otimes E\left[u_{n-r+1}, \ldots, u_{n}\right], d\right], \\
d\left(u_{i}\right) & =w_{i}(\xi),
\end{aligned}
$$

and so if $w_{n-r+1}(\xi)=\cdots=w_{n}(\xi)=0$ we see that

$$
E_{2}=H^{*}(B) \otimes E\left[u_{n-r+1}, \ldots, u_{n}\right] \text {, }
$$

where $\operatorname{deg} u_{i}=(-1, i)$. Thus we see that $E_{2}=E_{\infty}$ and that $E_{2}$ is a free $H^{*}(B)$ module, so $H^{*}(E)$ is a free $H^{*}(B)$ module and hence $\xi$ is totally $\nsim 0$.

The reverse implication is trivial.

If $\xi$ is totally $\sim 0$ then the hypotheses of Theorem 3.1 hold with $J=(0)$ and hence we conclude

Corollary 4.6. $H^{*}(E)=U_{H^{*}(B)}\left(F^{-1}\right)$.

This example was first considered by Massey-Peterson [11].

Now it may happen that $\xi$ possesses a cross section $\sigma: B \rightarrow E$. Suppose that this is so. We then have an exact sequence

$$
0 \longrightarrow H^{*}(B) \stackrel{p^{*}}{\longrightarrow} F^{-1} \longrightarrow E_{\infty}^{-1, *} \longrightarrow 0
$$

which is split by an $\mathscr{A}(2)$ map $s^{*}$ obtained by restricting $\sigma^{*}: H^{*}(E) \rightarrow H^{*}(B)$ to $F^{-1} \subset H^{*}(E)$ and hence we obtain

COROLlaRY 4.7. If $\xi$ has a cross section then

where

$$
H^{*}(E)=H^{*}(B) \otimes U(X)
$$

$$
X=\operatorname{Tor}_{H^{*}\left(B_{S O}(n)\right)}^{-*}\left(H^{*}(B), H^{*}\left(B_{S O(n-r)}\right)\right) \text {. }
$$

The $\mathscr{A}(2)$ module structure on $X$ is discussed in [11].

Example 4. An example of Massey and Peterson. Suppose that $X$ is a simply connected space and that $x_{1}, \ldots, x_{n} \in H^{*}\left(X ; Z_{2}\right)$ is an ESP-sequence. We can form a fibre space construction that kills off these classes as follows. Choose maps

$$
f_{i}: X \rightarrow K\left(Z_{2}, \operatorname{dim} x_{i}\right)
$$

representing $x_{i}$, i.e., such that $f_{i}^{*}\left(\dot{\eta}_{i}\right)=x_{i}$ where $\dot{\eta}_{i} \in H^{*}\left(Z_{2}, \operatorname{dim} x_{i}, Z_{2}\right)$ is the fundamental class. 
Let $B_{0}=\mathbf{X}_{i=1}^{n} K\left(Z_{2}, \operatorname{dim} x_{i}\right)$ and define the obvious map $f: X \rightarrow B_{0}$. Assume that $\operatorname{dim} x_{i}>0$ and so $B_{0}$ is simply connected. Form the diagram

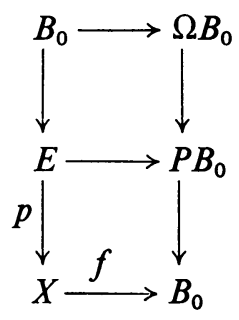

Definition. Classes $x_{1}, \ldots, x_{n} \in H^{*}(X)\left(Z_{2}\right.$ coefficients $)$ are called representable if there exists a map

$$
f: Y \rightarrow X
$$

for some space $Y$ with $\operatorname{ker} f^{*}=\left(x, \ldots, x_{n}\right)$.

Corollary 4.8 (Massey-Peterson). If $x_{1}, \ldots, x_{n}$ are representable then $H^{*}(E)$ $=U_{R}\left(F^{-1}\right)$, where $R=H^{*}(B) /\left(x_{1}, \ldots, x_{n}\right)$ as $R-\mathscr{A}(2)$ algebras.

Proof. We first note that since $x_{1}, \ldots, x_{n}$ are representable, the ideal $\left(x_{1}, \ldots, x_{n}\right)$ is an $\mathscr{A}(2)$ ideal. The result now follows from Serre's calculations [16].

We note that Corollary 3.7 shows that in this case the above construction "exactly" kills off the classes $x_{1}, \ldots, x_{n}$ since $\operatorname{ker} p^{*}=\left(x_{1}, \ldots, x_{n}\right)$. Note also that for this to be so the classes $x_{1}, \ldots$ need not be representable but must generate an $\mathscr{A}(2)$ ideal.

EXAmple 5. Secondary characteristic classes. The previous examples lead rather naturally to the study of secondary characteristic classes as found in [14].

Let us build a universal example for bundles with fibre $V_{n, r}$ that are totally nonhomologous to zero. So let $B_{1} \rightarrow B_{\text {SO(n) }}$ be a fibre space constructed as in Example 4 to kill off the classes $w_{n-r+1}, \ldots, w_{n} \in H^{*}\left(B_{S O(n)}\right)$. The cohomology of $B_{1}$ can be computed as in Example 4.

Now it is easy to see that given a fibre bundle $\xi=\left(E, p, B, V_{n, r}, S O(n)\right)$ that is totally $\nsim 0$ then there exists a bundle map (not unique)

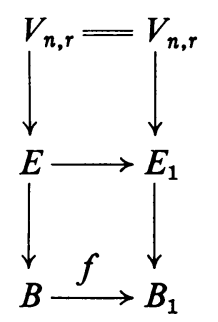

where $\xi_{1}=\left(E_{1}, p_{1}, B_{1}, V_{n, r}, S O(n)\right)$ is obtained from the universal bundle $\xi_{0}$ by pullback along $f_{1}: B_{1} \rightarrow B_{\text {SO }(n)}$. 
CoROllaRY 4.9. $H^{*}(E)=H^{*}(B) \otimes_{H^{*}\left(B_{1}\right)} H^{*}\left(E_{1}\right)$ as an $\mathscr{A}(2)$ algebra.

Proof. Example 2.

Thus a knowledge of the cohomology of $B_{1}$ and $E_{1}$ would be essential to computing the cohomology of $E$. Using Example 4 and Serre's calculations [16] we can show

COROLLARY 4.10. There is a filtration of $H^{*}\left(B_{1}\right)$ with

$$
E^{0} H^{*}\left(B_{1}\right)=P\left[w_{2}, \ldots, w_{n-r}\right] \otimes E\left[\left\{x_{i, r}\right\}\right]
$$

where $n-r+1 \leqq i \leqq n$ and I ranges over all admissible sequences of excess less than $i$.

$$
\operatorname{deg} x_{i, I}=(-1, i+\operatorname{deg} I) .
$$

Thus if we choose $\theta_{i, I} \in H^{*}\left(B_{1}\right)$ corresponding to $x_{i, I} \in E^{0} H^{*}\left(B_{1}\right)$ we can define "exotic" or secondary characteristic classes for totally $\nsim 0$ bundles $\xi$ by $\theta_{i, I}(\xi)$ $=\left\{f^{*}\left(\theta_{i, I}\right) \mid f\right.$ ranges over all classifying maps $f: B \rightarrow B_{1}$ for $\left.\xi\right\}$.

COROLlaRY 4.11. $H^{*}(E)$ is determined as an $\mathscr{A}(2)$ algebra by the classes $\theta_{i, I}(\xi), w_{i}(\xi)$.

Proof. We note that the monomials of exponent 0,1 in $\theta_{i, I}$ are a basis for $H^{*}\left(B_{1}\right)$ over $P\left[w_{2}, \ldots, w_{n}\right]$ and so knowledge of the multiplicative map $f^{*}$ on the classes $w_{i}, \theta_{i, 1}$ determines $f^{*}$ and hence the algebra $H^{*}(E)$.

Example 6. Stable two-stage Postnikov systems. By a generalized EilenbergMacLane space we shall mean a space $B_{0}=\mathbf{X} K\left(\Pi_{i}, n_{i}\right)$ where the $\Pi_{i}$ are finitely generated abelian groups.

Suppose that $B_{0}$ is a simply connected generalized Eilenberg-MacLane space (GEM for short). A two-stage Postnikov system is a diagram

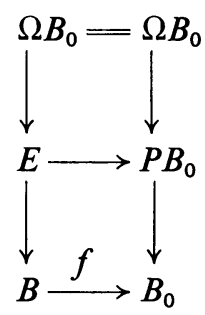

where $\Omega B_{0} \rightarrow P B_{0} \rightarrow B_{0}$ is the path space fibration, and $\Omega B_{0} \rightarrow E \rightarrow B$ is the fibration induced by $f: B \rightarrow B_{0}$. We denote the above diagram by $\mathscr{E}$.

Now $B$ and $B_{0}$ have natural $H$-space structures induced by the group operation in $\Pi_{i}$. We will say that $\mathscr{E}$ is stable if $f: B \rightarrow B_{0}$ is a map of $H$-spaces where $B$ and $B_{0}$ have $H$-space structures equivalent to the natural one.

Corollary 4.12. If $\mathscr{E}$ is a stable two-stage Postnikov system and $B$ has no factors of the form $K(Z, 1), K\left(Z_{2}^{n}, 1\right), n>1$, then using $Z_{2}$ coefficients $H^{*}(E)=U_{R}\left(F^{-1}\right)$ as $R-\mathscr{A}(2)$ algebras where $R=H^{*}(E) /\left((\mathrm{im}) \bar{f}^{*}\right)$. 
Proof. Clearly it suffices to show that $\left((\mathrm{im}) \bar{f}^{*}\right)$ is a Borel ideal in $H^{*}(B)$. The result then follows by Corollary 3.5 and Corollary 3.6. To show this is a Borel ideal we shall need the following two facts about Hopf algebras

FACT A. If $\Gamma$ is a connected Hopf algebra and $\Lambda$ is a Hopf subalgebra of $\Gamma$, then $\Gamma$ is a free $\Lambda$ module (see [12, Proposition 4.4]).

FACT B. If $\Omega$ is a graded commutative connected Hopf algebra over $Z_{2}$ then as an algebra

$$
\Omega=P\left[x_{1}, \ldots\right] \otimes P\left[y_{1}, \ldots\right] /\left(y_{1}^{r_{1}}, \ldots\right)
$$

where the $r_{i}$ are powers of 2 [12, Proposition 7.8].

From Serre's calculations it follows that $H^{*}\left(B_{0}\right)$ and $H^{*}(B)$ are polynomial algebras. Since $f: B \rightarrow B_{0}$ is a map of $H$-spaces it follows that $f^{*}: H^{*}\left(B_{0}\right) \rightarrow H^{*}(B)$ is a map of Hopf algebras. Therefore $\operatorname{im} f^{*} \subset H^{*}(B)$ is a Hopf subalgebra. Thus by Fact $\mathrm{B} \operatorname{im} f^{*}=P\left[x_{1}, \ldots\right]$ and by Fact $\mathrm{A} H^{*}(B)$ is a free $\operatorname{im} f^{*}$ module. Therefore by Proposition 1.1 (im) $\bar{f}^{*}$ generates a Borel ideal in $H^{*}(B)$.

This is the same problem originally considered by Kristensen in [8]. Our results seem somewhat simpler.

Our notion of stability differs somewhat from that employed in the past so let us put it in perspective. Massey-Peterson note that $H^{*}(B)=U(X)$ and $H^{*}\left(B_{0}\right)=U(Y)$ for suitable $X$ and $Y$. They say that $\mathscr{E}$ is stable in $f^{*}(Y) \subset X$. Note that $f^{*}: Y \rightarrow X$ completely determines $f^{*}: U(Y) \rightarrow U(X)$. Note also that in the stable case $f^{*}$ is a map of Hopf algebras (stable in their sense) and hence the proof of Corollary 4.12 applies.

Stability in our sense certainly implies stability in the sense of Massey-Peterson since $X$ and $Y$ are the primitive elements in $H^{*}(B)$ and $H^{*}\left(B_{0}\right)$ respectively and a map of Hopf algebras preserves primitive elements. The converse is false as shown by an example of Massey-Peterson.

Using slightly different techniques we have obtained a generalization of this example to $Z_{p}$ coefficients, $p$ a prime. This will appear in [17].

\section{REFERENCES}

1. J. F. Adams, On the non-existence of elements of Hopf invariant one, Ann. of Math. (2) 72 (1960), 20-104.

2. P. Baum, Cohomology of homogeneous spaces, (to appear).

3. A. Borel, Sur l'homologie et la cohomologie des groupes de lie compacts connexes, Amer. J. Math. 76 (1954), 273-342.

4. - Sur la cohomologie des espaces fibrés principaux, Ann. of Math. (2) 57 (1953), 115-207.

5. H. Cartan and S. Eilenberg, Homological algebra, Princeton Univ. Press, Princeton, N. J., 1956.

6. S. Eilenberg and J. C. Moore, Homology and fibrations. I, II, III, Comment. Math. Helv. 40 (1966), 199-236; (to appear).

7. S. Eilenberg and N. Steenrod, Foundations of algebraic topology, Princeton Univ. Press, Princeton, N. J., 1952. 
8. L. Kristensen, On the cohomology of two-stage Postnikov systems, Acta Math. 107 (1962), 73-123.

9. - On the cohomology of spaces with two non-vanishing homotopy groups, Math. Scand. 12 (1963), 83-105.

10. S. MacLane, Homology, Academic Press, New York; Springer-Verlag, Berlin, 1963.

11. W. S. Massey and F. P. Peterson, Cohomology of certain fibre spaces. I, Topology 4 (1965), 47-65.

12. J. Milnor and J. C. Moore, The structure of Hopf algebras, Ann. of Math. 81 (1965), 211-264.

13. J. C. Moore, Algèbre homologique et des espaces classifiants, Séminaire Cartan et Moore 1959/1960, Exposé 7, Ecole Norm. Sup. Paris.

14. F. P. Peterson and N. Stein, Secondary characteristic classes, Ann. of Math. (2) 76 (1962), 510-523.

15. J.-P. Serre, Homologie singulière des espaces fibrés. Applications, Ann. of Math. (2) 54 (1951), 425-505.

16. - Cohomologie modulo 2 des complexes d'Eilenberg-MacLane, Comment. Math. Helv. 27 (1953), 198-232.

17. L. Smith, Cohomology of stable two stage Postnikov systems, Illinois J. Math. 11 (1967), 310-329.

18. N. Steenrod and D. B. A. Epstein, Cohomology operations, Annals of Mathematics Studies, No. 50, Princeton University Press, Princeton, N. J., rev. ed. 1962.

19. N. E. Steenrod, Products of cocycles and extensions of mappings, Ann. of Math. (2) 48 (1947), 290-320.

20. J. Tate, Homology of Noetherian rings and of local rings, Illinois J. Math. 1 (1957), 14-27.

21. S. Eilenberg and J. C. Moore, Limits and spectral sequences, Topology 1 (1962), 1-24.

\author{
YALE UNIVERSITY, \\ New Haven, Connecticut \\ Princeton UNIVERSITY, \\ Princeton, New Jersey
}

